\title{
BOUNDARY REGULARITY OF ROTATING VORTEX PATCHES
}

\author{
TAOUFIK HMIDI, JOAN MATEU, AND JOAN VERDERA
}

\begin{abstract}
We show that the boundary of a rotating vortex patch (or V-state, in the terminology of Deem and Zabusky) is $C^{\infty}$ provided the patch is close to the bifurcation circle in the Lipschitz norm. The rotating patch is also convex if it is close to the bifurcation circle in the $C^{2}$ norm. Our proof is based on Burbea's approach to $V$-states.
\end{abstract}

\section{Contents}

1. Introduction

2. Burbea's approach to V-states

3. Existence of $m$-fold V-states

3.1. The spaces $X$ and $Y$

3.2. $F(\lambda, f)$ is in $C^{1+\alpha}(\mathbb{T})$

3.3. Real Fourier coefficients

3.4. $\mathrm{F}$ is Gateaux differentiable

3.5. Continuity of $D_{f} F(\lambda, f)$

3.6. Kernel and range of $D_{f} F(\lambda, 0)$

3.7. $m$-fold symmetry

3.8. Kirchhoff's ellipses

4. Boundary smoothness of rotating vortex patches References

\section{INTRODUCTION}

The motion of a two dimensional inviscid incompressible fluid is governed by Euler equations

$$
\left\{\begin{array}{l}
\partial_{t} v(z, t)+(v \cdot \nabla v)(z, t)=-\nabla p(z, t), \quad z \in \mathbb{C}, \quad t>0 \\
\operatorname{div} v=0 \\
v(z, 0)=v_{0}(z)
\end{array}\right.
$$

where $v(z, t)$ is the velocity field at the point $(z, t) \in \mathbb{C} \times \mathbb{R}_{+}$and $p$ is the pressure, which is a scalar function. The operators $v \cdot \nabla$ and div are defined by

$$
v \cdot \nabla=v_{1} \partial_{1}+v_{2} \partial_{2} \quad \text { and } \quad \operatorname{div} v=\partial_{1} v_{1}+\partial_{2} v_{2} .
$$

The velocity field $v$ is divergence free because the fluid is incompressible. In dimension two the vorticity is given by the scalar $\omega=\partial_{1} v_{2}-\partial_{2} v_{1}$. One can recover the velocity from the vorticity by means of the Biot-Savart law. Indeed, identifying $v=\left(v_{1}, v_{2}\right)$ with $v_{1}+i v_{2}$ and performing a simple calculation one gets

$$
2 \partial v=i \omega, \quad \text { with } \quad \partial:=\partial_{z}=\frac{1}{2}\left(\partial_{1}-i \partial_{2}\right) .
$$


Since $z \mapsto \frac{1}{\pi \bar{z}}$ is the fundamental solution of the complex operator $\partial$ we get the BiotSavart law

$$
v(z, t)=\frac{i}{2 \pi} \int_{\mathbb{C}} \frac{\omega(\zeta, t)}{\bar{z}-\bar{\zeta}} d A(\zeta), \quad z \in \mathbb{C},
$$

$d A$ being planar Lebesgue measure. Taking curl in the first equation of the system (1) one obtains the vorticity equation

$$
\left\{\begin{array}{l}
\partial_{t} \omega+v \cdot \nabla \omega=0 \\
\omega(z, 0)=\omega_{0}(z)
\end{array}\right.
$$

where $\omega_{0}$ denotes the initial vorticity and $v$ is given by the Biot-Savart law (2). Equation (31) simply means that the vorticity is constant along particle trajectories. Under mild smoothness assumptions the Euler system is equivalent to the vorticity formulation (3)(21). A convenient reference for these results is [BM, Chapter 2].

It is a deep fact, known as Yudovich Theorem, that the vorticity equation has a unique global solution in the weak sense when the initial vorticity $\omega_{0}$ lies in $L^{1} \cap L^{\infty}$. See for instance [BM, Chapter 8]). A vortex patch is the solution of (3) with initial condition the characteristic function of a bounded domain $D_{0}$. Since the vorticity is transported along trajectories, we get that $\omega(z, t)=\chi_{D_{t}}(z)$, where $D_{t}=X\left(D_{0}, t\right)$ is the image of $D_{0}$ by the flow. Recall that the flow $X$ is the solution of the nonlinear integral equation

$$
X(z, t)=z+\int_{0}^{t} v(X(z, \tau), \tau) d \tau, \quad z \in \mathbb{C}, t \geq 0 .
$$

In the special case where $D_{0}$ is the open unit disc the vorticity is radial and thus we get a steady flow. In particular, $D_{t}=D_{0}, t \geq 0$, and the particle trajectories are circles centered at the origin. A remarkable fact discovered by Kirchoff is that when the initial condition is the characteristic function of an ellipse centered at the origin, then the domain $D_{t}$ is a rotation of $D_{0}$. Indeed, $D_{t}=e^{i t \Omega} D_{0}$, where the angular velocity $\Omega$ is determined by the semi-axis $a$ and $b$ of the initial ellipse through the formula $\Omega=a b /(a+b)^{2}$. See for instance [BM, p.304] and [L, p.232].

A rotating vortex patch or $\mathrm{V}$-state is a domain $D_{0}$ such that if $\chi_{D_{0}}$ is the initial condition of the vorticity equation, then the region of vorticity 1 rotates with constant angular velocity around its center of mass, which we assume to be the origin. In other words, $D_{t}=e^{i t \Omega} D_{0}$ or, which is the same, the vorticity at time $t$ is given by

$$
\omega(z, t)=\chi_{D_{0}}\left(e^{-i t \Omega} z\right), \quad z \in \mathbb{C}, \quad t>0 .
$$

Here the angular velocity $\Omega$ is a positive number associated with $D_{0}$.

To the best of our knowledge the ellipse is the only $\mathrm{V}$-state for which a closed formula is known. Deem and Zabusky [DZ] wrote an equation for the V-states and solved it numerically. They found V-states with $m$-fold symmetry for each integer $m \geq 2$. A domain is said to be $m$-fold symmetric if is invariant under a rotation of angle $2 \pi / m$. One may view such a domain as being the union of $m$ leaves each of which can be obtained from a given one by rotating it of an angle of the form $p(2 \pi / m)$, for some integer $p$. It is extremely interesting to look at the pictures of $m$-fold symmetric V-states in [WOZ] : one can see domains with smooth boundary, which evolve with certain associated parameters to produce in the limit domains whose boundaries have corners at right angles.

Burbea [B] gave a mathematical proof of the existence of $m$-fold symmetric $V$-states using bifurcation from the circle solution. His approach is, in Aref's words, elegant and deep [A, p. 346]. It is indeed so. He finds an equation for the V-states and then uses conformal mapping to rewrite the equation in a functional analytic framework in which 
bifurcation theory can be applied. Unfortunately the proof has a gap, which occurs when the space to which bifurcation theory should be applied is set up. The suggestion made in [B, p.8] of using the standard Hardy space does not work. One reason is that the operator $Q(f)$ in [B, p.8] involves one derivative of $f$ and functions in the Hardy space do not necessarily have derivatives. Another reason is that one needs a space which guarantees that small analytic perturbations of the identity in the space are conformal. A space which fulfils the preceding requirements is the space of Lipschitz functions. However, for technical reasons, the space of Lipschitz functions is not suitable for our purposes and has to be replaced by the smaller space of functions with first order derivatives satisfying a Hölder condition of order $\alpha, 0<\alpha<1$. The reader will find an exposition of Burbea's approach in section 2 and a complete proof of the existence of $m$ - fold symmetric V-states in section 3. It is our impression that this beautiful and striking theorem deserves to be more widely known than it appears to be now. In section 4 we prove our main result, which states that if a bifurcated $V$-state is close enough to the circle in the Lipschitz sense then its boundary is of class $C^{\infty}$. We also show that a bifurcated V-state is convex if it is close enough to the circle in the $C^{2}$ norm. There is a dark zone, where the $\mathrm{V}$-state has boundary of class $C^{1+\alpha}$ for some positive $\alpha$ but it is not close enough to the circle, in which the actual smoothness properties of the boundary are unknown. Also the nature of the singularities of the "limiting" V-states of Wu, Overman and Zabusky ([WOZ]) are not well understood at all.

We adhere to the convention of denoting by $C$ a constant independent of the relevant parameters under consideration. The constant may change its actual value at different occurrences.

Added on October 11, 2014. While working on the manuscript [HHMV] we realized that some of the proofs presented in this paper can be substantially simplified. For instance, subsection 3.3 on real Fourier coefficients has been now reduced to just a remark (see page 11). Moreover, most of the technical work which has to be done when checking that Crandall-Rabinowitz's Theorem can be applied is simplified if one works with the differentiated form of Burbea's equation, which is (8) in page 5 of [HHMV]. See also (47). We have kept here the original proofs. The alternative simpler arguments can be found in [HHMV]. The reader should be aware that in [HHMV] one works with domains with two boundary components and so the presentation has to be adapted to the easier simply connected case.

\section{BurbeA's APPROACH TO V-STATES}

We first derive an equation for simply connected vortex patches which have smooth boundary for all times. Consider two parametrizations of the boundary $\partial D_{t}$ of the patch at time $t$, say $z(\alpha, t)$ and $\eta(\beta, t)$, and assume that they are proper in the sense that they establish a homeomorphism between the interval of definition of the parameters with the extremes identified and $\partial D_{t}$. Assume also that they are continuously differentiable as functions of the parameter and time. Then there exists a change of parameters $\alpha(\beta, t)$ such that $\eta(\beta, t)=z(\alpha(\beta, t), t)$ for all $\beta$ and $t$ and so we have

$$
\frac{\partial \eta}{\partial t}(\beta, t)=\frac{\partial z}{\partial \alpha}(\alpha, t) \frac{\partial \alpha(\beta, t)}{\partial t}+\frac{\partial z}{\partial t}(\alpha, t) .
$$

Since $\frac{\partial z}{\partial \alpha}(\alpha, t)$ is a tangent vector to the boundary at the point $z(\alpha, t)$ and $\frac{\partial \alpha}{\partial t}(\beta, t)$ is a scalar we conclude that

$$
\frac{\partial \eta}{\partial t}(\beta, t) \cdot \vec{n}=\frac{\partial z}{\partial t}(\alpha, t) \cdot \vec{n}
$$


where $\vec{n}$ is the exterior unit normal vector at the point $z(\alpha, t)=\eta(\beta, t)$ and the dot stands for scalar product in $\mathbb{R}^{2}=\mathbb{C}$. Thus the quantity in (44) does not depend on the parametrization and represents the speed of the boundary in the normal direction. On the other hand $v(z(\alpha, t), t) \cdot \vec{n}$ is the normal component of the velocity of a particle which is located at the point $z(\alpha, t)$ at time $t$. Since the boundary is advected by the velocity $v$ we get the equation

$$
\frac{\partial z}{\partial t}(\alpha, t) \cdot \vec{n}=v(z(\alpha, t), t) \cdot \vec{n}
$$

which describes the motion of the boundary of the patch.

Now we introduce the stream function

$$
\psi(z, t)=\left(\frac{1}{2 \pi} \log |\cdot| * \chi_{D_{t}}\right)(z) .
$$

Clearly $\bar{\partial} \log |z|^{2}=1 / \bar{z}$, where $\bar{\partial}=\partial / \partial \bar{z}$. Hence

$$
2 i \bar{\partial} \psi(z, t)=\frac{i}{2 \pi}\left(\frac{1}{\bar{z}} * \chi_{D_{t}}\right)(z)=v(z, t)
$$

and

$$
\begin{aligned}
(v \cdot \vec{n})(z(\alpha, t), t) & =\left(\nabla^{\perp} \psi \cdot \vec{n}\right)(z(\alpha, t), t) \\
& =-(\nabla \psi \cdot \vec{\tau})(z(\alpha, t), t) \\
& =-\frac{d \psi}{d s}(z(\alpha, t), t) .
\end{aligned}
$$

where $\vec{\tau}$ is the unit tangent vector and $s$ is the arc-length parameter of the curve $\partial D_{t}$. Therefore (5) becomes

$$
\frac{d \psi}{d s}(z(\alpha, t))=-\frac{\partial z(\alpha, t)}{\partial t} \cdot \vec{n}
$$

Assume now that the patch rotates with angular velocity $\Omega$. Let $z_{0}(\alpha)$ be a proper continuously differentiable parametrization of $\partial D_{0}$ and set $z(\alpha, t)=e^{i \Omega t} z_{0}(\alpha)$, which is a proper parametrization of $\partial D_{t}$. Then

$$
\frac{\partial z}{\partial t}(\alpha, t)=i \Omega z(\alpha, t)
$$

and (7) becomes

$$
\frac{d \psi}{d s}(z(\alpha, t), t)=-i \Omega z(\alpha, t) \cdot \vec{n}=\Omega z(\alpha, t) \cdot \vec{\tau} .
$$

Taking $\alpha=s$, the arc-length parameter on $\partial D_{0}$, we obtain

$$
\frac{d}{d s}|z(s, t)|^{2}=2 \operatorname{Re}(\overline{z(s, t)} \vec{\tau})=2 z(s, t) \cdot \vec{\tau}
$$

so that, by (8),

$$
\frac{d \psi}{d s}(z(s, t), t)=\frac{\Omega}{2} \frac{d}{d s}|z(s, t)|^{2}
$$

and integrating with respect to $s$ yields, for a certain constant $c(t)$ depending on $t$,

$$
\psi(z, t)=\frac{\Omega}{2}|z|^{2}+c(t), \quad z \in \partial D_{t} .
$$

Since the steps can be reversed this is the equation of $\mathrm{V}$-states.

The goal now is to use conformal mappings to "parametrize" V-states. For that one needs to modify the preceding equation to get a form more amenable to the use of 
analytic functions. Fix $t$ and take derivatives in (9) with respect to $s$ on $\partial D_{t}$. We just get a restatement of (8), that is,

$$
2 \operatorname{Re}\left(\frac{\partial \psi}{\partial z}(z(s, t), t) z^{\prime}(s, t)\right)=\operatorname{Re}\left(\overline{\Omega(s, t)} z^{\prime}(s, t)\right)
$$

where the prime means derivative with respect to $s$. By the generalized Cauchy formula (which follows from a direct application of Green-Stokes) one has

$$
\bar{z}=\frac{1}{2 \pi i} \int_{\partial D_{t}} \frac{\bar{\zeta}}{\zeta-z} d \zeta+\frac{1}{\pi} \int_{D_{t}} \frac{d A(\zeta)}{z-\zeta}, \quad z \in D_{t} .
$$

Taking the $\partial=\partial / \partial z$ derivative in (6) and applying (11)

$$
4 \frac{\partial \psi}{\partial z}(z, t)=\frac{1}{\pi} \int_{D_{t}} \frac{d A(\zeta)}{z-\zeta}=\bar{z}-\frac{1}{2 \pi i} \int_{\partial D_{t}} \frac{\bar{\zeta}}{\zeta-z} d \zeta, \quad z \in D_{t} .
$$

The first identity above implies that $z \mapsto \frac{\partial \psi}{\partial z}(z, t)$ extends continuously to the closed domain $\overline{D_{t}}$, since the Cauchy integral of a bounded compactly supported function is quasi Lipschitz (its modulus of continuity is $O(\delta|\log \delta|), \delta<1 / 2$ ). Thus the same happens to the Cauchy integral of the function $\bar{\zeta}$ on $\partial D_{t}$. Hence (10) becomes, with $\lambda=1-2 \Omega$,

$$
\operatorname{Re}\left(\lambda \bar{z} z^{\prime}(s, t)-\frac{1}{2 \pi i} \int_{\partial D_{t}} \frac{\bar{\zeta}}{\zeta-z} d \zeta z^{\prime}(s, t)\right)=0, \quad z \in \partial D_{t}
$$

where the integral over $\partial D_{t}$ for $z \in \partial D_{t}$ should be understood as the limit as $w \in D_{t}$ tends to $z$ of the corresponding integral for $w$.

Integrating with respect to $s$ on $\partial D_{t}$ we conclude that, for some constant $c(t)$ depending on $t$,

$$
\lambda|z|^{2}+2 \operatorname{Re} \frac{1}{2 \pi i} \int_{\partial D_{t}} \bar{\zeta} \log \left(1-\frac{z}{\zeta}\right) d \zeta=c(t), \quad z \in \partial D_{t}
$$

Remember that the origin belongs to $D_{t}$ and thus, for each $\zeta \in \partial D_{t}$, there exists a branch of the logarithm of $1-z / \zeta$ in $D_{t} \backslash\{\zeta\}$ taking the value 0 at $z=0$. Also fixed $z \in \partial D_{t}$ there exists a branch of the logarithm of $1-z / \zeta$ in $\mathbb{C}_{\infty} \backslash D_{t} \cup\{z\}$ taking the value 0 at $\zeta=\infty$. Therefore the integral in (12) exists for each $z \in \partial D_{t}$ and defines a continuous function on $\partial D_{t}$. Later on we will differentiate this integral with respect to $z$ on $\partial D_{t}$.

Notice that the equation (12) is invariant by rotations and dilations. Consequently, using the fact $D_{t}=e^{i t \Omega} D_{0}$ and performing a change of variables equation (12) reduces to

$$
\lambda|z|^{2}+2 \operatorname{Re} \frac{1}{2 \pi i} \int_{\partial D_{0}} \bar{\zeta} \log \left(1-\frac{z}{\zeta}\right) d \zeta=c, \quad z \in \partial D_{0} .
$$

We have used the fact $c(t)$ does not depend on $t$ since the left hand side of (13) is independent on the time variable. Equation (13) is Burbea's equation for simply connected V-states.

We introduce now conformal mappings. Let $E_{D_{0}}=\mathbb{C}_{\infty} \backslash \overline{D_{0}}$ be the exterior of $D_{0}$ and $E_{\triangle}=\mathbb{C}_{\infty} \backslash \bar{\triangle}$ the exterior of the unit disc $\triangle=\{z:|z|<1\}$. Let $\Phi$ be a conformal mapping of $E_{\Delta}$ onto $E_{D_{0}}$ preserving the point at $\infty$. This mapping can be expanded in $E_{\Delta}$ as

$$
\Phi(z)=a\left(z+\sum_{n \geq 0} \frac{a_{n}}{z^{n}}\right)
$$

for some complex number $a$. Making a rotation in the independent variable $z$ we can assume that $a$ is a positive number and then dilating the domain we can further assume 
that $a=1$. Following Burbea we change variables in (13) setting $z=\Phi(w)$ and $\zeta=\Phi(\tau)$. Then (13) becomes

$$
\lambda|\Phi(w)|^{2}+2 \operatorname{Re} \frac{1}{2 \pi i} \int_{\mathbb{T}} \overline{\Phi(\tau)} \log \left(1-\frac{\Phi(w)}{\Phi(\tau)}\right) \Phi^{\prime}(\tau) d \tau=c, \quad w \in \mathbb{T} .
$$

Recall that we are assuming $\partial D_{0}$ to have rectifiable boundary. It is then a classical result that $\Phi$ can be extended continuously to the unit circle $\mathbb{T}=\{z \in \mathbb{C}:|z|=1\}$ and the extension is absolutely continuous, so that $\Phi^{\prime}(w)$ exists for almost all $w \in \mathbb{T}$ and the resulting function is in $L^{1}(\mathbb{T})$ (see $[\mathrm{P}]$ ). Later on we will work with a $\Phi$ whose extension to $\mathbb{T}$ is of class $C^{1+\alpha}(\mathbb{T})$ so that $\Phi^{\prime}$ will be Hölder continuous of order $\alpha$ on $\mathbb{T}$. To simplify the notation, set

$$
\sigma(\Phi)(w)=\frac{1}{2 \pi i} \int_{\mathbb{T}} \overline{\Phi(\tau)} \log \left(1-\frac{\Phi(w)}{\Phi(\tau)}\right) \Phi^{\prime}(\tau) d \tau, \quad w \in \mathbb{T}
$$

and

$$
m(\Phi, \lambda)=\frac{1}{2 \pi} \int_{\mathbb{T}}\left(\lambda|\Phi(w)|^{2}+2 \operatorname{Re} \sigma(\Phi)(w)\right)|d w|,
$$

where $|d w|$ denotes the length measure on the circle. Hence (14) is

$$
\lambda|\Phi(w)|^{2}+2 \operatorname{Re} \sigma(\Phi)(w)-m(\Phi, \lambda)=0, \quad w \in \mathbb{T} .
$$

It is more convenient to set $\Phi(z)=z+f(z)$ with $f$ analytic on $E_{\Delta}$ and define the operator

$$
S(f)(w)=\sigma(\Phi)(w) .
$$

That this is a good point of view is confirmed by the fact that

$$
S(0)(w)=\frac{1}{2 \pi i} \int_{\mathbb{T}} \log \left(1-\frac{w}{\tau}\right) \frac{d \tau}{\tau}=0, \quad w \in \mathbb{T},
$$

because the integrand is analytic on $E_{\triangle}$ and has a double zero at $\infty$. Thus (17) is satisfied for $\Phi(z)=z$ and each $\lambda$. Now define

$$
F(\lambda, f)(w):=\lambda|w+f(w)|^{2}+2 \operatorname{Re} S(f)(w)-m(\mathrm{I}+f, \lambda), \quad w \in \mathbb{T},
$$

where $I$ stands for the identity function. Clearly Burbea's equation can be rewritten as

$$
F(\lambda, f)=0 \text {. }
$$

One has

$$
F(\lambda, 0)=0, \quad \lambda \in \mathbb{R}
$$

which simply says that the disc satisfies Burbea's equation (13) for each $\lambda$. Burbea's idea at this point is to apply bifurcation theory in order to prove the existence of $\mathrm{m}$ fold V-states. We will apply in the next section Crandall-Rabinowitz's Theorem whose original statement in [CR, p.325] is included below for the reader's convenience. For a linear mapping $L$ we let $N(L)$ and $R(L)$ stand for the kernel and range of $L$ respectively. If $Y$ is a vector space and $R$ is a subspace, then $Y / R$ denotes the quotient space.

Crandall-Rabinowitz's Theorem. Let $X, Y$ be Banach spaces, $V$ a neighborhood of 0 in $X$ and

$$
F:(-1,1) \times V \rightarrow Y
$$

have the properties

(a) $F(t, 0)=0$ for any $|t|<1$.

(b) The partial derivatives $F_{t}, F_{x}$ and $F_{t x}$ exist and are continuous.

(c) $N\left(F_{x}(0,0)\right)$ and $Y / R\left(F_{x}(0,0)\right)$ are one-dimensional. 
(d) $F_{t x}(0,0) x_{0} \notin R\left(F_{x}(0,0)\right)$, where

$$
N\left(F_{x}(0,0)\right)=\operatorname{span}\left\{x_{0}\right\} .
$$

If $Z$ is any complement of $N\left(F_{x}(0,0)\right)$ in $X$, then there is a neighborhood $U$ of $(0,0)$ in $\mathbf{R} \times X$, an interval $(-a, a)$, and continuous functions $\varphi:(-a, a) \rightarrow \mathbf{R}, \psi:(-a, a) \rightarrow Z$ such that $\varphi(0)=0, \psi(0)=0$ and

$$
F^{-1}(0) \cap U=\left\{\left(\varphi(\xi), \xi x_{0}+\xi \psi(\xi)\right):|\xi|<a\right\} \cup\{(t, 0):(t, 0) \in U\} .
$$

\section{Existence of $m$-FOLd V-STATES}

In this section we will apply Crandall-Rabinowitz's Theorem to prove the existence of $m$-fold V-states for each integer $m \geq 2$. For $m=2$ one recovers the Kirchoff ellipses. As a by-product of this formalism we get a Hölderian boundary regularity result for the $\mathrm{V}$-states which are close to a point of the bifurcation set $\left\{(\lambda, f)=\left(\frac{1}{m}, 0\right), m=2,3, \ldots\right\}$. Later we will see how to establish the $C^{\infty}$ regularity of the boundary of these V-states by using hidden smoothing effects of the nonlinear equation (17). First, we establish the following result.

Theorem 1. Given $0<\alpha<1$ and $m=2,3, \ldots$ there exists a curve of $m$-fold rotating vortex patches with boundary of class $C^{1+\alpha}$ bifurcating at the circle solution.

More precisely, there exist $a>0$ and continuous functions $\lambda:(-a, a) \rightarrow \mathbf{R}, \psi:(-a, a) \rightarrow$ $C^{1+\alpha}(\mathbb{T})$ satisfying $\lambda(0)=1 / m, \psi(0)=0$, such that the Fourier series of $\psi(\xi)$ is of the form

$$
\psi(\xi)(w)=a_{2 m-1}(\xi) \bar{w}^{2 m-1}+\cdots+a_{n m-1}(\xi) \bar{w}^{n m-1}+\ldots, \quad w \in \mathbb{T},
$$

and

$$
F\left(\lambda(\xi), \xi \bar{w}^{m-1}+\xi \psi(\xi)(w)\right)=0, \quad \xi \in(-a, a)
$$

The mapping

$$
\Phi_{\xi}(z)=z\left(1+\xi \frac{1}{z^{m}}+\xi a_{2 m-1}(\xi) \frac{1}{z^{2 m}}+\cdots+\xi a_{n m-1}(\xi) \frac{1}{z^{n m}}+\ldots\right)
$$

is conformal and of class $C^{1+\alpha}$ on $\mathbb{C} \backslash \Delta$, and the complement $D_{\xi}$ of $\Phi_{\xi}(\mathbb{C} \backslash \Delta)$ is an $m$-fold rotating vortex patch.

The proof of this theorem requires some lengthly work, which will be presented in several steps. We start by introducing the spaces $X$ and $Y$ and then we will check all the assumptions of Crandall-Rabinowitz's Theorem.

3.1. The spaces $X$ and $Y$. The choice of the spaces $X$ and $Y$ is a key point, which was overlooked in $[\mathrm{B}]$. Before giving the complete description of these spaces we need first to recall the definition of the Hölder spaces $C^{n+\alpha}(\Omega)$. Let $\Omega$ be a nonempty open set of $\mathbb{R}^{d}$ and $0<\alpha<1$. We denote by $C^{\alpha}(\Omega)$ the space of continuous functions $f$ such that

$$
\|f\|_{C^{\alpha}(\Omega)}:=\|f\|_{L^{\infty}}+\sup _{x \neq y \in \Omega} \frac{|f(x)-f(y)|}{|x-y|^{\alpha}}<\infty,
$$

where $\|f\|_{L^{\infty}}$ stands for the supremum norm of $f$ on $\Omega$. More generally, for a non-negative integer $n$ the Hölder space $C^{n+\alpha}(\Omega)$ consists of those functions of class $C^{n}$ whose $n$-th order derivatives are Hölder continuous with exponent $\alpha$ in $\Omega$. It is equipped with the norm

$$
\|f\|_{C^{n+\alpha}(\Omega)}=\|f\|_{L^{\infty}}+\sum_{|\gamma|=n}\left\|\partial^{\gamma} f\right\|_{C^{\alpha}(\Omega)} .
$$


We will also make use of the space $C^{1+\alpha}(\mathbb{T})$ which is the set of continuously differentiable functions $f$ on the unit circle $\mathbb{T}$ whose derivatives satisfy a Hölder condition of order $\alpha$, endowed with the norm

$$
\|f\|_{C^{1+\alpha}(\mathbb{T})}=\|f\|_{L^{\infty}}+\left\|\frac{d f}{d w}\right\|_{\alpha},
$$

where $\|\cdot\|_{\alpha}$ is the usual Lipschitz semi-norm of order $\alpha$

$$
\|g\|_{\alpha}=\sup _{x \neq y \in \mathbb{T}} \frac{|g(x)-g(y)|}{|x-y|^{\alpha}}
$$

We define in a similar way the spaces $C^{n+\alpha}(\mathbb{T})$, for each positive integer $n$ and $\left.\alpha \in\right] 0,1[$. A word on the operator $d / d w$ is in order. Any function $f: \mathbb{T} \rightarrow \mathbb{R}$ can be identified with a $2 \pi$-periodic function $g: \mathbb{R} \rightarrow \mathbb{R}$ via the formula

$$
f(w)=g(\theta), \quad w=e^{i \theta} .
$$

Therefore for a smooth function $f$ we get

$$
\frac{d f}{d w}=-i e^{-i \theta} g^{\prime}(\theta)
$$

It will be more convenient in the sequel to work with $d / d w$ instead of $d / d \theta$. Since they differ only by a smooth factor it really makes no difference. Notice that we have the identity

$$
\frac{d\{\bar{f}\}}{d w}=-\frac{1}{w^{2}} \frac{\overline{d f}}{d w} .
$$

On the other hand, if we denote by $C_{2 \pi}^{1+\alpha}(\mathbb{R})$ the subspace of $C^{1+\alpha}(\mathbb{R})$ consisting of $2 \pi$ periodic functions, then we can identify $C^{1+\alpha}(\mathbb{T})$ with $C_{2 \pi}^{1+\alpha}(\mathbb{R})$ and

$$
\|g\|_{\alpha} \approx\|f\|_{\alpha} .
$$

Let $C_{a}^{1+\alpha}\left(\Delta^{c}\right)$ be the space of analytic functions on $\mathbb{C}_{\infty} \backslash \bar{\triangle}$ whose derivatives satisfy a Hölder condition of order $\alpha$ up to $\mathbb{T}$. This is also the space of functions in $C^{1+\alpha}(\mathbb{T})$ whose Fourier coefficients of positive frequency vanish.

The space $X$ is defined as

$$
X=\left\{f \in C^{1+\alpha}(\mathbb{T}) ; f(w)=\sum_{n=0}^{\infty} a_{n} \bar{w}^{n}, w \in \mathbb{T}, a_{n} \in \mathbb{R}, n \geq 0\right\}
$$

and coincides with the subspace of $C_{a}^{1+\alpha}\left(\Delta^{c}\right)$ consisting of those functions in $C_{a}^{1+\alpha}\left(\Delta^{c}\right)$ whose boundary values have real Fourier coefficients. Later on we will modify appropriately the space $X$ to get $m$-fold symmetry, but for now it is simpler and clearer to work with this $X$. The reader will understand later why we require the $a_{n}$ to be real. For the time being we just comment on the geometric meaning of this requirement. If we set, for $f \in X, \Phi(z)=z+f(z)$ and $\Phi$ happens to be conformal on $\mathbb{C}_{\infty} \backslash \bar{\triangle}$, then the complement in $\mathbb{C}_{\infty}$ of the closure of $\Phi\left(\mathbb{C}_{\infty} \backslash \bar{\triangle}\right)$ is a simply connected domain $D$ symmetric with respect to the real axis. Conversely, if one starts with a bounded simply connected domain $D$ symmetric with respect to the real axis and $\Phi$ is the conformal mapping of the complement of the closed unit disc onto the complement of $\bar{D}$, then the coefficients in the expansion of $\Phi$ as a power series in $1 / z$ are real. Now, if one is given a domain $D$ with an axis of symmetry containing the origin, after a rotation one can assume that this axis is the real line. Domains with $m$-fold symmetry have an axis of symmetry, so it is not really restrictive for our purposes to work with functions with real Fourier coefficients. 
Let $V$ stand for the open ball with center 0 and radius 1 in $C_{a}^{1+\alpha}\left(\Delta^{c}\right)$. If $f$ is in $V$ then the function $\Phi(z)=z+f(z)$ is analytic on $\{z:|z|>1)$ and is injective there. For, by the maximum principle,

$$
\left\|\frac{d f}{d w}\right\|_{L^{\infty}\left(\Delta^{c}\right)}=\sup \left\{\frac{|f(z)-f(w)|}{|z-w|}:|z| \geq 1,|w| \geq 1, z \neq w\right\}:=\delta<1,
$$

and hence

$$
|\Phi(z)-\Phi(w)| \geq|z-w|-|f(z)-f(w)| \geq(1-\delta)|z-w|, \quad|z| \geq 1,|w| \geq 1 .
$$

For $f \in V$ define $S(f)$ as in (18) and (15), where $\Phi(z)=z+f(z)$. The definition makes sense precisely because $\Phi$ is injective and thus a branch of the logarithm of $1-\Phi(w) / \Phi(\tau)$ can be defined taking the value 1 at $\infty$ (that is, as $\tau \rightarrow \infty$ ), as we argued after (13)). We define now a function $F(\lambda, f)$ on $\mathbb{R} \times V$ by

$$
F(\lambda, f)(w)=\lambda|w+f(w)|^{2}+2 \operatorname{Re} S(f)(w)-m(\operatorname{Id}+f, \lambda),
$$

where $S(f)$ is as in (18) and $m(\operatorname{Id}+f, \lambda)$ as in (16). This is the function $F$ to which we will apply Crandall-Rabinowitz's Theorem.

We define now the space $Y$ as the subspace of $C^{1+\alpha}(\mathbb{T})$ consisting of real-valued functions with zero integral and real Fourier coefficients. More precisely,

$$
Y:=\left\{g \in C^{1+\alpha}(\mathbb{T}): g(w)=\sum_{0 \neq n \in \mathbb{Z}} a_{n} w^{n}, w \in \mathbb{T} \text { and } a_{n}=a_{-n} \in \mathbb{R}, n>0\right\} .
$$

Since we have subtracted the mean in (23) it is clear that $F(\lambda, f)$ is a real-valued function with zero integral. To show that $F$ maps $X$ into $Y$ it remains to show that $F(\lambda, f)$ belongs to $C^{1+\alpha}(\mathbb{T})$ and that its Fourier coefficients are real.

3.2. $F(\lambda, f)$ is in $C^{1+\alpha}(\mathbb{T})$. Since $F(\lambda, f)$ has zero integral its norm in the space $C^{1+\alpha}(\mathbb{T})$ is equivalent to the $C^{\alpha}(\mathbb{T})$ norm of its derivative $w \mapsto \frac{d F}{d w}(\lambda, f)(w)$. Now, since $C^{1+\alpha}(\mathbb{T})$ is an algebra the problem reduces to showing that $w \mapsto \frac{d S(f)}{d w}(w)$ belongs to $C^{\alpha}(\mathbb{T})$. For that we need to compute the derivative of $S(f)(w)$ with respect to $w$ and this is done in the next lemma. Recall that $\Phi: \mathbb{T} \rightarrow \mathbb{C}$ is bilipschitz (into the image) if for a positive constant $C$ one has

$$
C^{-1}|\tau-\omega| \leq|\Phi(\tau)-\Phi(\omega)| \leq C|\tau-\omega|, \quad \tau, \omega \in \mathbb{T} .
$$

Lemma 1. For any bilipschitz function $\Phi: \mathbb{T} \rightarrow \mathbb{C}$ of class $C^{1}(\mathbb{T})$ we have

$$
\frac{d}{d w} S(f)(w)=-\Phi^{\prime}(w)\left(\frac{\overline{\Phi(w)}}{2}+p . v \cdot \frac{1}{2 \pi i} \int_{\mathbb{T}} \frac{\overline{\Phi(\tau)} \Phi^{\prime}(\tau)}{\Phi(\tau)-\Phi(w)} d \tau\right), \quad w \in \mathbb{T} .
$$

Proof. The proof of this lemma consists in computing the derivative with respect to $w$ in the sense of distributions by integrating against a test function on $\mathbb{T}$. When integrating by parts we will get $-\Phi^{\prime}(w)$ times the principal value integral in (25) plus a "boundary term" $-\Phi^{\prime}(w) \overline{\Phi(w)} / 2$, which is due to the fact that the logarithm in the definition of $S(f)$ is not continuous on the diagonal.

An alternative argument goes as follows. Bring the integral defining $S(f)$ on the $C^{1}$ Jordan curve $\Gamma=\Phi(\mathbb{T})$ to obtain the function

$$
\sigma(z)=\frac{1}{2 \pi i} \int_{\Gamma} \bar{\zeta} \log \left(1-\frac{z}{\zeta}\right) d \zeta, \quad z \in \Gamma
$$


Think of $\sigma$ as an analytic function of $z \in D$, the domain enclosed by $\Gamma$. Its derivative is

$$
-\frac{1}{2 \pi i} \int_{\Gamma} \frac{\bar{\zeta}}{\zeta-z} d \zeta=-\bar{z}-\frac{1}{\pi} \int_{D} \frac{1}{\zeta-z} d A(\zeta), \quad z \in D
$$

where the identity is the generalized Cauchy formula for the function $\bar{z}$. The integral on $D$ in the right-hand side is a quasi-Lipschitz function, as the Cauchy integral of a bounded function, and hence the left-hand side extends continuously up to the boundary. Call this extension $-C(\bar{\zeta})(z), z \in \Gamma$, where the notation refers to "boundary values of the Cauchy integral of the function $\bar{\zeta}$ ". By Plemelj's formula (see, for example, $[\mathrm{V}$, p. 143])

$$
C(\bar{\zeta})(z)=\frac{\bar{z}}{2}+\text { p.v. } \frac{1}{2 \pi i} \int_{\Gamma} \frac{\bar{\zeta}}{\zeta-z} d \zeta
$$

We conclude that $\sigma$ is differentiable on $\Gamma$ and its derivative with respect to $z$ is given by minus the expression in (26). Changing variables to return to the unit circle and applying the chain rule we get (25).

Now everything is reduced to checking that the the principal value integral in (25) satisfies a Hölder condition of order $\alpha$. This can be done rather easily in at least two ways. The first consists in considering the operator

$$
T g(w)=\text { p.v. } \frac{1}{2 \pi i} \int_{\mathbb{T}} \frac{g(\tau)}{\Phi(\tau)-\Phi(w)} d \tau, \quad w \in \mathbb{T},
$$

and showing that $T$ maps boundedly $C^{\alpha}(\mathbb{T})$ into itself. This can be achieved by applying the T(1) - Theorem for Hölder spaces of Wittmann [W, Theorem 2.1, p.584] (see also Ga]). Before stating Witmann's result recall that the maximal singular integral of $g$ is

$$
T^{*}(g)(w)=\sup _{\epsilon>0}\left|\int_{\mathbb{T} \backslash D(w, \epsilon)} \frac{g(\tau)}{\Phi(\tau)-\Phi(w)} d \tau\right|, \quad w \in \mathbb{T},
$$

$D(w, \epsilon)$ being the disc centered at $w$ of radius $\epsilon$. Wittmann's $T(1)-$ Theorem asserts in our context that the $C^{\alpha}(\mathbb{T})$ boundedness of $T$ follows by checking that the kernel is "standard", that $T^{*}(1)$ is bounded and that the operator $T$ applied to the constant function 1 lies in $C^{\alpha}(\mathbb{T})$. That the kernel is standard means in the situation we are considering that

$$
\left|\frac{1}{\Phi(\tau)-\Phi(w)}\right| \leq \frac{C}{|\tau-w|}, \quad \tau, w \in \mathbb{T}
$$

and

$$
\left|\frac{d}{d w}\left(\frac{1}{\Phi(\tau)-\Phi(w)}\right)\right| \leq \frac{C}{|\tau-w|^{2}}, \quad \tau, w \in \mathbb{T}
$$

which are clearly satisfied because $\Phi$ is bilipschitz on its domain. To compute $T(1)(w), w \in$ $\mathbb{T}$, denote by $\gamma_{\epsilon}, \epsilon>0$, the arc which is the intersection of the circle centered at $w$ of radius $\epsilon$ and the complement of the open unit disc, with the counter-clockwise orientation. Let $\mathbb{T}_{\epsilon}$ be the closed Jordan curve consisting of the arc $\gamma_{\epsilon}$ followed by the part of the unit circle at distance from $w$ not less than $\epsilon$. We claim that

$$
\begin{aligned}
T(1)(w) & =\lim _{\varepsilon \rightarrow 0}\left(\frac{1}{2 \pi i} \int_{\mathbb{T}_{\varepsilon}} \frac{\tau-w}{\Phi(\tau)-\Phi(w)} \frac{d \tau}{\tau-w}-\frac{1}{2 \pi i} \int_{\gamma_{\epsilon}} \frac{\tau-w}{\Phi(\tau)-\Phi(w)} \frac{d \tau}{\tau-w}\right) \\
& =1-\frac{1}{2 \Phi^{\prime}(w)}
\end{aligned}
$$


The integral over $\mathbb{T}_{\epsilon}$ is 1 since the integrand is analytic as a function of $\tau$ on the exterior of the unit disc and we have

$$
\lim _{|\tau| \rightarrow \infty} \frac{\tau-w}{\Phi(\tau)-\Phi(w)}=1
$$

The limit as $\epsilon \rightarrow 0$ of the integral over $\gamma_{\epsilon}$ is

$$
\frac{1}{\Phi^{\prime}(w)} \lim _{\epsilon \rightarrow 0} \int_{\gamma_{\epsilon}} \frac{d \tau}{\tau-w}=\frac{\pi i}{\Phi^{\prime}(w)}
$$

and so (30) is proven. From the assumption $\Phi \in C^{1+\alpha}(\mathbb{T})$ combined with (30) we obtain that $T(1) \in C^{\alpha}(\mathbb{T})$. It is also clear that $T^{*}(1)$ is bounded on $\mathbb{T}$ from the argument above.

For future reference we record now the following identity, whose proof is similar to that just described of (30),

$$
\mathrm{p} . \mathrm{v} \cdot \frac{1}{2 \pi i} \int_{\mathbb{T}} \frac{\Phi^{\prime}(\tau)}{\Phi(\tau)-\Phi(w)} d \tau=\frac{1}{2} .
$$

The second argument to show that the principal value integral in (25) satisfies a Hölder condition of order $\alpha$ consists in changing variables $\zeta=\Phi(\tau)$ and $z=\Phi(w)$ and pass to a principal value integral on the $C^{1+\alpha}$ Jordan curve $\Gamma=\Phi(\mathbb{T})$. The kernel of the operator one obtains is the Cauchy kernel and the integration is with respect to $d \zeta$. This operator sends $C^{\alpha}(\Gamma)$ into itself on curves satisfying a mild regularity assumption called Ahlfors regularity. This can be proved by standard arguments and we prefer to omit the lengthly calculations and inequalities involved.

3.3. Real Fourier coefficients. We intend to show that if $f \in X$ then $F(\lambda, f)$ has real Fourier coefficients. This is very simple once we notice that an integrable function $g$ on $\mathbb{T}$ has real Fourier coefficients if and only if $g(\bar{w})=\overline{g(w)}$. Examining the definition of $F(\lambda, f)$ in (23) we conclude that only the term $S(f)(w)$ has to be considered, the others having clearly real Fourier coefficients. The identity $S(f)(\bar{w})=\overline{S(f)(w)}$ follows from the obvious change of variables $z=\bar{\tau}$.

3.4. $\mathbf{F}$ is Gateaux differentiable. We show here that $F$ is Gateaux differentiable and in the next subsection we will check that the directional derivatives are continuous. This will show that $F$ is continuously differentiable on its domain $\mathbb{R} \times V$. In proving differentiability properties of $F(\lambda, f)$ we can consider only the first two terms in (20) because the third, $m(I+f, \lambda)$, is a mean of these two terms. Denote by $G(\lambda, f)$ the sum of the first two terms in (20).

There is no problem with the partial derivative with respect to $\lambda$ because the dependence on $\lambda$ is linear. We get

$$
D_{\lambda} F(\lambda, f)(w)=|w+f(w)|^{2}
$$

which is obviously continuous with respect to $f$ in the topology of $X$.

Take now $h \in X$ and compute the derivative of $G(\lambda, f)$ with respect to $f$ in the direction $h$, that is,

$$
\begin{aligned}
D_{f} G(\lambda, f)(h) & =\left.\frac{d}{d t} G(\lambda, f+t h)\right|_{t=0} \\
& =2 \lambda \operatorname{Re}(\Phi \bar{h})+\left.2 \operatorname{Re} \frac{d}{d t} S(f+t h)\right|_{t=0} .
\end{aligned}
$$


The computation of $\left.\frac{d}{d t} S(f+t h)\right|_{t=0}$ yields the four terms below,

$$
\begin{aligned}
\frac{d}{d t} S\left(f+\left.t h\right|_{t=0}(w)\right. & =\frac{1}{2 \pi i} \int_{\mathbb{T}} \overline{h(\tau)} \log \left(1-\frac{\Phi(w)}{\Phi(\tau)}\right) \Phi^{\prime}(\tau) d \tau \\
& +\quad+\frac{1}{2 \pi i} \int_{\mathbb{T}} \overline{\Phi(\tau)} \log \left(1-\frac{\Phi(w)}{\Phi(\tau)}\right) h^{\prime}(\tau) d \tau \\
& +\frac{1}{2 \pi i} \int_{\mathbb{T}} \overline{\Phi(\tau)} \frac{h(w)-h(\tau)}{\Phi(\tau)-\Phi(w)} \Phi^{\prime}(\tau) d \tau \\
& +\frac{1}{2 \pi i} \int_{\mathbb{T}} \overline{\Phi(\tau)} h(\tau) \Phi^{\prime}(\tau) d \tau \\
& :=A(f, h)(w)+B(f, h)(w)+C(f, h)(w)+D(f, h),
\end{aligned}
$$

where the last identity is the definition of the functions $A, B, C$ and $D$. Notice that $D$ is independent of $w$.

We proceed now to prove that $A(f, h) \in C^{1+\alpha}(\mathbb{T})$. For the estimate of the absolute value of $A(f, h)(w)$ we can assume without loss of generality that $w=1$. Computing the derivative of $\log (1-\Phi(1) / \Phi(\tau))$ with respect to $\tau$ in $|\tau|>1$ and using the fundamental theorem of calculus we see that

$$
\log \left(1-\frac{\Phi(1)}{\Phi(\tau)}\right)=-\int_{1}^{\infty} \frac{\Phi(1) \Phi^{\prime}(t \tau)}{(\Phi(t \tau)-\Phi(1)) \Phi(t \tau)} \tau d t, \quad 1 \neq \tau, \quad|\tau| \geq 1
$$

The preceding identity is very useful in estimating the integrals containing a logarithmic term, as we will see below. We proceed now to estimate from below the factors in the denominator of the fraction inside the integral in (34). The function $\Phi(\tau) / \tau$ is analytic on $\mathbb{C} \backslash \bar{\Delta}$ and takes the value 1 at $\infty$. Hence, by the maximum principle and recalling that $f \in V$, we obtain

$$
1-\|f\|_{L^{\infty}(\mathbb{T})} \leq \frac{|\Phi(\tau)|}{|\tau|} \leq 1+\|f\|_{L^{\infty}(\mathbb{T})}, \quad|\tau| \geq 1
$$

and

$$
|\Phi(\tau)-\Phi(1)| \geq\left(1-\left\|f^{\prime}\right\|_{L^{\infty}(\mathbb{T})}\right)|\tau-1|, \quad|\tau| \geq 1
$$

Therefore, by (34), (35) and (36) we get for $\tau \in \mathbb{T} \backslash\{1\}$,

$$
\begin{aligned}
\left|\log \left(1-\frac{\Phi(1)}{\Phi(\tau)}\right)\right| & \leq|\Phi(1)|\left\|\Phi^{\prime}\right\|_{L^{\infty}\left(\Delta^{c}\right)} \int_{1}^{\infty} \frac{d t}{|\Phi(t \tau)-\Phi(1)||\Phi(t \tau)|} \\
& \leq \frac{\left(1+\|f\|_{L^{\infty}(\mathbb{T})}\right)\left(1+\left\|f^{\prime}\right\|_{L^{\infty}(\mathbb{T})}\right)}{\left(1-\|f\|_{L^{\infty}(\mathbb{T})}\right)\left(1-\left\|f^{\prime}\right\|_{L^{\infty}(\mathbb{T})}\right)} \int_{1}^{\infty} \frac{d t}{t|t \tau-1|} .
\end{aligned}
$$

We split the interval of integration in the last integral above in three subintervals : $(1,1+|\tau-1|),(1+|\tau-1|, 3)$ and $(3, \infty)$. In the first we notice that for $t \geq 1$ and $|\tau|=1$ we have the elementary inequality

$$
|t \tau-1|=|t-\bar{\tau}|=|t-\tau| \geq|1-\tau|
$$

and so

$$
\int_{1}^{1+|\tau-1|} \frac{d t}{t|t \tau-1|} \leq 1
$$

The integral on the second interval can be estimated straightforwardly as follows

$$
\int_{1+|\tau-1|}^{3} \frac{d t}{t|t \tau-1|} \leq \int_{\substack{1+|\tau-1| \\ 12}}^{3} \frac{d t}{t-1}=\log \frac{2}{|\tau-1|}
$$


Finally

$$
\int_{3}^{\infty} \frac{d t}{t|t \tau-1|} \leq \int_{3}^{\infty} \frac{d t}{t(t-1)}=\log \frac{3}{2}
$$

Therefore, collecting the preceding inequalities,

$$
\left|\log \left(1-\frac{\Phi(1)}{\Phi(\tau)}\right)\right| \leq C(f)(1+|\log | \tau-1||), \quad \tau \in \mathbb{T},
$$

where $C(f)$ is a constant depending only on the $C^{1}(\mathbb{T})$ norm of $f$. Integration in $\tau$ on $\mathbb{T}$ readily yields

$$
\|A(f, h)\|_{\infty} \leq C_{1}(f)\|h\|_{\infty} .
$$

The next step is to estimate the uniform norm and the Lipschitz semi-norm of order $\alpha$ of the function $w \mapsto \frac{d}{d w} A(f, h)(w)$. As in Lemma 1 one has

$$
\frac{d}{d w} A(f, h)(w)=-\Phi^{\prime}(w)\left(\frac{\overline{h(w)}}{2}+\text { p.v. } \frac{1}{2 \pi i} \int_{\mathbb{T}} \frac{\overline{h(\tau)} \Phi^{\prime}(\tau)}{\Phi(\tau)-\Phi(w)} d \tau\right), \quad w \in \mathbb{T},
$$

and the only difficulty lies in estimating the $C^{\alpha}(\mathbb{T})$ norm of the principal value integral. But this has already been done in subsection 3.2. Therefore $A(f, h) \in C^{1+\alpha}(\mathbb{T})$.

The proof that the term $B(f, h)$ belongs to $C^{1+\alpha}(\mathbb{T})$ is basically the same. The expression for the derivative is

$$
\frac{d}{d w} B(f, h)(w)=-\Phi^{\prime}(w)\left(\frac{\overline{\Phi(w)} h^{\prime}(w)}{2 \Phi^{\prime}(w)}+\text { p.v. } \frac{1}{2 \pi i} \int_{\mathbb{T}} \frac{h^{\prime}(\tau) \overline{\Phi(\tau)} d \tau}{\Phi(\tau)-\Phi(w)}\right)
$$

and one deals with the principal value integral as before, using the operator in (27).

The proof that the term $C(f, h)$ is in $C^{1+\alpha}(\mathbb{T})$ contains a little variation of the preceding argument. First, we observe that the quotient

$$
\frac{h(\tau)-h(w)}{\Phi(\tau)-\Phi(w)}
$$

takes continuously the value $h^{\prime}(w) / \Phi^{\prime}(w)$ on the diagonal of $\mathbb{T}$. Consequently, in computing the derivative of $C(f, h)(w)$ no boundary terms will arise and we get

$$
\begin{aligned}
\frac{d}{d w} C(f, h)(w) & =h^{\prime}(w) \text { p.v. } \frac{1}{2 \pi i} \int_{|\tau|=1} \frac{g(\tau)}{\Phi(\tau)-\Phi(w)} d \tau \\
& +\Phi^{\prime}(w) \text { p.v. } \frac{1}{2 \pi i} \int_{\mathbb{T}} \frac{h(w)-h(\tau)}{(\Phi(\tau)-\Phi(w))^{2}} g(\tau) d \tau
\end{aligned}
$$

where $g(\tau)=\overline{\Phi(\tau)} \Phi^{\prime}(\tau)$. The mapping properties of the operator $T$ in (27) take care of the principal value integral in the first term. We view the principal value integral in the second term as an operator $U$ acting on $g$. Its kernel is standard, because $\Phi$ is bilipschitz, and the action of $U$ on the constant function 1 is, as in (30),

$$
\begin{aligned}
U(1)(w) & =\lim _{\varepsilon \rightarrow 0} \int_{\mathbb{T}_{\varepsilon}} \frac{h(w)-h(\tau)}{(\Phi(\tau)-\Phi(w))^{2}} d \tau \\
& +\lim _{\varepsilon \rightarrow 0} \frac{1}{2 \pi i} \int_{\gamma_{\varepsilon}} \frac{h(\tau)-h(w)}{\tau-w}\left(\frac{\tau-w}{\Phi(\tau)-\Phi(w)}\right)^{2} \frac{d \tau}{\tau-w} \\
& =\frac{h^{\prime}(w)}{2 \Phi^{\prime}(w)^{2}} .
\end{aligned}
$$


In the integral on $\mathbb{T}_{\epsilon}$ the integrand is analytic on $\mathbb{C} \backslash \bar{\triangle}$ and has a double zero at $\infty$, and so the integral vanishes. The limit as $\epsilon$ tends to 0 of the integral on $\gamma_{\epsilon}$ is $i \pi$ times the limit as $\tau$ tends to $w$ of the quotients inside, that is, $h^{\prime}(w) / \Phi^{\prime}(w)^{2}$. Then $U(1) \in C^{\alpha}(\mathbb{T})$. The previous discussion also gives that $U^{*}(1)$ is bounded. Thus one can apply Wittmann's $T(1)$-Theorem (see the paragraph after (27)) and conclude that $U$ maps $C^{\alpha}(\mathbb{T})$ into itself. This completes the proof that $w \mapsto D_{f} F(\lambda, f)(h)(w) \in C^{1+\alpha}(\mathbb{T})$. On the other hand, it is clear that $D_{f} F(\lambda, f)(h)$ depends linearly on $h$. Therefore $F(\lambda, f)$ is Gateaux differentiable at any point $(\lambda, f) \in \mathbb{R} \times V$.

3.5. Continuity of $D_{f} F(\lambda, f)$. In this subsection we prove that the mapping $D_{f} F(\lambda, f)$ is continuous as a function of $f \in V$ taking values in the space of bounded linear operators from $X$ into $Y$. In particular this shows that $F(\lambda, f)$ is continuously differentiable in the Frechet sense. What one has to do is the following. Fix $f \in V$ and show an inequality of the type

$$
\left\|D_{f} F(\lambda, f)(h)-D_{g} F(\lambda, g)(h)\right\|_{C^{1+\alpha}(\mathbb{T})} \leq C_{1+\alpha}(f)\|f-g\|_{C^{1+\alpha}(\mathbb{T})}\|h\|_{C^{1+\alpha}(\mathbb{T})}
$$

for $g \in X$ close enough to $f$. Here we denote by $C_{1+\alpha}(f)$ a constant depending on the norm $\|f\|_{C^{1+\alpha}}$. By (32) and (33) this amounts to prove similar inequalities for $A(f, h), B(f, h)$ and $C(f, h)$ in place of $D_{f} F(\lambda, f)$. For instance,

$$
\|A(f, h)-A(g, h)\|_{C^{1+\alpha}(\mathbb{T})} \leq C_{1+\alpha}(f)\|f-g\|_{C^{1+\alpha}(\mathbb{T})}\|h\|_{C^{1+\alpha}(\mathbb{T})}
$$

for $g \in X$ close to $f$. We start with the estimate of the uniform norm of the difference

$$
\|A(f, h)-A(g, h)\|_{\infty} \leq C_{1}(f)\|f-g\|_{C^{1}(\mathbb{T})}\|h\|_{L^{\infty}(\mathbb{T})} .
$$

To prove the uniform estimate above it is enough to consider the point $w=1 \mathrm{in} \mathbb{T}$. Take $g \in V$ close to $f$ and set $\Psi(\tau)=\tau+g(\tau),|\tau| \geq 1$. We can easily check that

$$
\begin{aligned}
A(f, h)(1)-A(g, h)(1) & =\frac{1}{2 \pi i} \int_{\mathbb{T}} \overline{h(\tau)} \log \left(1-\frac{\Phi(1)}{\Phi(\tau)}\right)\left(\Phi^{\prime}(\tau)-\Psi^{\prime}(\tau)\right) d \tau \\
& +\frac{1}{2 \pi i} \int_{\mathbb{T}} \overline{h(\tau)} \Psi^{\prime}(\tau)\left(\log \left(1-\frac{\Phi(1)}{\Phi(\tau)}\right)-\log \left(1-\frac{\Psi(1)}{\Psi(\tau)}\right)\right) d \tau \\
(40) \quad & \mathcal{I}_{1}(f, g)(h)+\mathcal{I}_{2}(f, g)(h) .
\end{aligned}
$$

To estimate the first term $\mathcal{I}_{1}(f, g)(h)$ we use (37),

$$
\begin{aligned}
\left|\mathcal{I}_{1}(f, g)(h)\right| & \leq \frac{1}{2 \pi}\|h\|_{\infty}\left\|f^{\prime}-g^{\prime}\right\|_{\infty} \int_{\mathbb{T}}\left|\log \left(1-\frac{\Phi(1)}{\Phi(\tau)}\right)\right||d \tau| \\
& \leq C(f)\|h\|_{\infty}\left\|f^{\prime}-g^{\prime}\right\|_{\infty} .
\end{aligned}
$$

To treat the second term $\mathcal{I}_{2}(f, g)(h)$, we use the identity (34) which yields

$$
\mathcal{K}(\tau)=\int_{1}^{\infty}\left(\frac{\Phi(1) \Phi^{\prime}(t \tau)}{(\Phi(t \tau)-\Phi(1)) \Phi(t \tau)}-\frac{\Psi(1) \Psi^{\prime}(t \tau)}{(\Psi(t \tau)-\Psi(1)) \Psi(t \tau)}\right) \tau d t, \quad \tau \in \mathbb{T} \backslash\{1\},
$$

where

$$
\mathcal{K}(\tau):=\log \left(1-\frac{\Psi(1)}{\Psi(\tau)}\right)-\log \left(1-\frac{\Phi(1)}{\Phi(\tau)}\right)
$$


By elementary algebraic computations one gets

$$
\begin{aligned}
\mathcal{K}(\tau) & =\int_{1}^{\infty} \frac{(\Phi(1)-\Psi(1)) \Phi^{\prime}(t \tau)}{(\Phi(t \tau)-\Phi(1)) \Phi(t \tau)} \tau d t+\Psi(1) \int_{1}^{\infty} \frac{\Phi^{\prime}(t \tau)-\Psi^{\prime}(t \tau)}{(\Phi(t \tau)-\Phi(1)) \Phi(t \tau)} \tau d t \\
& +\Psi(1) \int_{1}^{\infty} \Psi^{\prime}(t \tau) \frac{\Psi(t \tau)-\Phi(t \tau)}{(\Phi(t \tau)-\Phi(1)) \Phi(t \tau) \Psi(t \tau)} \tau d t \\
& +\Psi(1) \int_{1}^{\infty} \frac{\Psi^{\prime}(t \tau)}{\Psi(t \tau)}\left(\frac{1}{\Phi(t \tau)-\Phi(1)}-\frac{1}{\Psi(t \tau)-\Psi(1)}\right) \tau d t \\
& :=\sum_{j=1}^{4} \mathcal{K}_{j}(\tau) .
\end{aligned}
$$

Coming back to the identity (34), the first term $\mathcal{K}_{1}(\tau)$ takes the form

$$
\mathcal{K}_{1}(\tau)=-\frac{\Phi(1)-\Psi(1)}{\Phi(1)} \log \left(1-\frac{\Phi(1)}{\Phi(\tau)}\right) .
$$

It follows according to (37) that

$$
\left|\mathcal{K}_{1}(\tau)\right| \leq C_{1}(f)\|f-g\|_{L^{\infty}(\mathbb{T})}(1+|\log | \tau-1||) .
$$

As in the proof of (37), we obtain

$$
\begin{aligned}
\left|\mathcal{K}_{2}(\tau)\right| & \leq|\Psi(1)||| \Phi^{\prime}-\Psi^{\prime} \|_{L^{\infty}\left(\Delta^{c}\right)} \int_{1}^{\infty} \frac{1}{|\Phi(t \tau)-1||\Phi(t \tau)|} d \tau \\
& \leq|\Psi(1)||| f^{\prime}-g^{\prime} \|_{L^{\infty}(\mathbb{T})} \int_{1}^{\infty} \frac{1}{|\Phi(t \tau)-1||\Phi(t \tau)|} d \tau \\
& \leq C_{1}(f)\left\|f^{\prime}-g^{\prime}\right\|_{L^{\infty}(\mathbb{T})}(1+|\log | \tau-1||) .
\end{aligned}
$$

Concerning the third term, we write

$$
\begin{aligned}
\left|\mathcal{K}_{3}(\tau)\right| & \leq|\Psi(1)|\left\|\Psi^{\prime}\right\|_{L^{\infty}\left(\Delta^{c}\right)}\|\Phi-\Psi\|_{L^{\infty}\left(\Delta^{c}\right)} \int_{1}^{\infty} \frac{1}{|\Phi(t \tau)-1||\Phi(t \tau)||\Psi(t \tau)|} d \tau \\
& \leq C_{1}(f)\|f-g\|_{L^{\infty}(\mathbb{T})}(1+|\log | \tau-1||) .
\end{aligned}
$$

To treat the last term $\mathcal{K}_{4}$ we use (36)

$$
\begin{aligned}
\left|\frac{1}{\Phi(t \tau)-\Phi(1)}-\frac{1}{\Psi(t \tau)-\Psi(1)}\right| & =\left|\frac{\{\Psi-\Phi\}(t \tau)-\{\Psi-\Phi\}(1)}{(\Phi(t \tau)-\Phi(1))(\Psi(t \tau)-\Psi(1))}\right| \\
& \leq 4 \frac{\left\|\Psi^{\prime}-\Phi^{\prime}\right\|_{L^{\infty}\left(\Delta^{c}\right)}|t \tau-1|}{|t \tau-1|^{2}} \\
& \leq 4 \frac{\left\|f^{\prime}-g^{\prime}\right\|_{L^{\infty}(\mathbb{T})}}{|t \tau-1|} .
\end{aligned}
$$

Consequently,

$$
\left|\mathcal{K}_{4}(\tau)\right| \leq C_{1}(f)\left\|f^{\prime}-g^{\prime}\right\|_{L^{\infty}(\mathbb{T})}(1+|\log | \tau-1||)
$$

Therefore,

$$
|\mathcal{K}(\tau)| \leq C_{1}(f)\|f-g\|_{C^{1}(\mathbb{T})}(1+|\log | \tau-1||), \quad \tau \in \mathbb{T} \backslash\{1\} .
$$

Hence, coming back to the definition of $\mathcal{I}_{2}(f, g)(h)$ in (40) and integrating in $\tau$

$$
\left|\mathcal{I}_{2}(f, g)(h)\right| \leq C_{1}(f)\|f-g\|_{C^{1}(\mathbb{T})} .
$$


Finally,

$$
|A(f, h)(1)-A(g, h)(1)| \leq C_{1}(f)\|h\|_{L^{\infty}(\mathbb{T})}\|f-g\|_{C^{1}(\mathbb{T})}
$$

which is (39).

Our next task is to estimate the difference

$$
\frac{d}{d w} A(f, h)(w)-\frac{d}{d w} A(g, h)(w)
$$

in $C^{\alpha}(\mathbb{T})$ for $g$ close to $f$ in $C^{1+\alpha}(\mathbb{T})$. The difficult term in the formula for the derivative of the function $A(f, h)(w)$ in (38) is the principal value integral

$$
\begin{aligned}
I(f, h)(w) & =\text { p.v. } \frac{1}{2 \pi i} \int_{\mathbb{T}} \frac{\overline{h(\tau)} \Phi^{\prime}(\tau)}{\Phi(\tau)-\Phi(w)} d \tau \\
& =\frac{1}{2 \pi i} \int_{\mathbb{T}} \frac{\overline{h(\tau)}-\overline{h(w)}}{\Phi(\tau)-\Phi(w)} \Phi^{\prime}(\tau) d \tau+\frac{1}{2} \overline{h(w)} \\
& :=J(f, h)(w)+\frac{1}{2} \overline{h(w)} .
\end{aligned}
$$

Thus the difference $I(f, h)(w)-I(g, h)(w)$ is

$$
\begin{aligned}
J(f, h)(w)-J(g, h)(w) & =\frac{1}{2 \pi i} \int_{\mathbb{T}} \frac{\overline{h(\tau)}-\overline{h(w)}}{\Phi(\tau)-\Phi(w)}\left(\Phi^{\prime}(\tau)-\Psi^{\prime}(\tau)\right) d \tau \\
& +\frac{1}{2 \pi i} \int_{\mathbb{T}}(\overline{h(\tau)}-\overline{h(w)}) \Psi^{\prime}(\tau)\left(\frac{1}{\Phi(\tau)-\Phi(w)}-\frac{1}{\Psi(\tau)-\Psi(w)}\right) d \tau \\
& :=\mathcal{T}_{1}(f, g)(w)+\mathcal{T}_{2}(f, g)(w) .
\end{aligned}
$$

Now we need an estimate for $\mathcal{T}_{1}(f, g)$ and $\mathcal{T}_{2}(f, g)$ in $C^{\alpha}(\mathbb{T})$. Both terms have the form

$$
T \chi(w)=\int_{\mathbb{T}} K(w, \tau) \chi(\tau) d \tau, \quad \chi \in L^{\infty}(\mathbb{T})
$$

where the kernel $K(w, \tau)$ and the function $\chi$ are

$$
K(w, \tau)=\frac{\overline{h(\tau)}-\overline{h(w)}}{\Phi(\tau)-\Phi(w)}, \quad \chi(\tau)=\Phi^{\prime}(\tau)-\Psi^{\prime}(\tau)
$$

in $\mathcal{T}_{1}(f, g)(w)$ and

$$
K(w, \tau)=\frac{\overline{h(\tau)}-\overline{h(w)}}{\Phi(\tau)-\Phi(w)}-\frac{\overline{h(\tau)}-\overline{h(w)}}{\Psi(\tau)-\Psi(w)}, \quad \chi(\tau)=\Psi^{\prime}(\tau)
$$

in $\mathcal{T}_{2}(f, g)(w)$.

Lemma 2. Assume that the kernel of the operator $T$ in (43) satisfies

(1) $K$ is measurable on $\mathbb{T} \times \mathbb{T}$ and

$$
|K(w, \tau)| \leq C_{0}, \quad w, \tau \in \mathbb{T} .
$$

(2) For each $\tau \in \mathbb{T}, w \mapsto K(w, \tau)$ is differentiable in $\mathbb{T} \backslash\{w\}$ and

$$
\left|\partial_{w} K(w, \tau)\right| \leq \frac{C_{0}}{|w-\tau|}, \quad w, \tau \in \mathbb{T}, w \neq \tau .
$$

Then

$$
\left|T \chi\left(w_{1}\right)-T \chi\left(w_{2}\right)\right| \leq C\|\chi\|_{L^{\infty}} C_{0}\left(1+\log \frac{1}{\left|w_{1}-w_{2}\right|}\right)\left|w_{1}-w_{2}\right|, \quad w_{1} \neq w_{2} \in \mathbb{T}
$$

for some constant $C$. In particular, $T \chi \in C^{\alpha}(\mathbb{T})$ for every $0<\alpha<1$. 
The proof of this lemma is simple and standard. For the details of a similar result the reader is referred to [MOV, p.419].

To get the desired estimates for $\mathcal{T}_{1}(f, g)$ and $\mathcal{T}_{2}(f, g)$ we just need to check that their kernels satisfy the hypothesis of Lemma 2. We deal first with $\mathcal{T}_{1}(f, g)$. From (36) with the point $1 \in \mathbb{T}$ replaced by an arbitrary $w \in \mathbb{T}$, one readily gets

$$
|K(w, \tau)| \leq C\left\|h^{\prime}\right\|_{L^{\infty}(\mathbb{T})}, \quad\left|\partial_{w} K(w, \tau)\right| \leq C\left\|h^{\prime}\right\|_{L^{\infty}(\mathbb{T})}|\tau-w|^{-1} .
$$

Therefore

$$
\begin{aligned}
\left\|\mathcal{T}_{1}(f, g)\right\|_{C^{\alpha}(\mathbb{T})} & \leq C\left\|h^{\prime}\right\|_{L^{\infty}(\mathbb{T})}\left\|\Psi^{\prime}-\Phi^{\prime}\right\|_{L^{\infty}(\mathbb{T})} \\
& \leq C\left\|h^{\prime}\right\|_{L^{\infty}(\mathbb{T})}\|f-g\|_{C^{1}(\mathbb{T})} .
\end{aligned}
$$

To estimate the kernel of $\mathcal{T}_{2}(f, g)$ we use $(\underline{36})$

$$
\begin{aligned}
|K(w, \tau)| & =\frac{|h(\tau)-h(w)||(\Phi-\Psi)(w)-(\Phi-\Psi)(\tau)|}{|\Phi(\tau)-\Phi(w)||\Psi(\tau)-\Psi(w)|} \\
& \leq C\left\|h^{\prime}\right\|_{L^{\infty}(\mathbb{T})}\left\|\Phi^{\prime}-\Psi^{\prime}\right\|_{L^{\infty}(\mathbb{T})} .
\end{aligned}
$$

The derivative of $K(w, \tau)$ with respect to $w$ can also be estimated easily :

$$
\begin{aligned}
\left|\partial_{w} K(w, \tau)\right| & \leq\left|h^{\prime}(w)\right| \frac{1}{\Phi(\tau)-\Phi(w)}-\frac{1}{\Psi(\tau)-\Psi(w)} \mid \\
& +|h(\tau)-h(w)|\left|\frac{\Phi^{\prime}(w)}{(\Phi(\tau)-\Phi(w))^{2}}-\frac{\Psi^{\prime}(w)}{(\Psi(\tau)-\Psi(w))^{2}}\right| \\
& \leq C\left\|h^{\prime}\right\|_{L^{\infty}(\mathbb{T})}\left\|\Phi^{\prime}-\Psi^{\prime}\right\|_{L^{\infty}(\mathbb{T})}\left(1+\left\|\Phi^{\prime}+\Psi^{\prime}\right\|_{L^{\infty}(\mathbb{T})}\right) \frac{1}{|\tau-w|} \\
& \leq C_{1}(f)\|h\|_{C^{1}(\mathbb{T})}\|f-g\|_{C^{1}(\mathbb{T})} \frac{1}{|\tau-w|} .
\end{aligned}
$$

This gives according to Lemma 2

$$
\left\|\mathcal{T}_{2}(f, g)\right\|_{C^{\alpha}(\mathbb{T})} \leq C_{1}(f)\|h\|_{C^{1}(\mathbb{T})}\|f-g\|_{C^{1}(\mathbb{T})} .
$$

Hence we get

$$
\|I(f, h)-I(g, h)\|_{C^{1+\alpha}(\mathbb{T})} \leq C_{1}(f)\|h\|_{C^{1}(\mathbb{T})}\|f-g\|_{C^{1}(\mathbb{T})}
$$

and, finally, gathering all previous estimates

$$
\|A(f, h)-A(g, h)\|_{C^{1+\alpha}(\mathbb{T})} \leq C_{1}(f)\|f-g\|_{C^{1+\alpha}(\mathbb{T})}\|h\|_{C^{1+\alpha}(\mathbb{T})} .
$$

This concludes the proof of the continuity of the term $A(f, h)(w)$ with respect to $f$.

The proof for the terms $B(f, h), C(f, h)$ and $D(f, h)$ given by (33) follows a similar pattern. We omit the details.

3.6. Kernel and range of $D_{f} F(\lambda, 0)$. In this subsection we study the kernel and the range of $D_{f} F(\lambda, 0)$. We also find the "eigenvalues", that is, the values of $\lambda$ for which the kernel of $D_{f} F(\lambda, 0)$ is not trivial. In fact, the dimension of the kernel for these particular values of $\lambda$ turns out to be 1 . Then we check that the range has codimension 1 , so that Crandall-Rabinowitz's Theorem can be applied.

Letting $f=0$ in (32) and (33) we obtain

$$
D_{f} F(\lambda, 0)(h)(w)=2 \lambda \operatorname{Re}(h(w) \bar{w})+2 \operatorname{Re}\left(\frac{1}{2 \pi i} \int_{\mathbb{T}} \overline{h(\tau)} \log \left(1-\frac{w}{\tau}\right) d \tau\right)-m(h),
$$

where $m(h)$ is the mean on $\mathbb{T}$ with respect to $|d w|$ of the sum of the first two terms in the right-hand side above. Hence $w \mapsto D_{f} F(\lambda, 0)(h)(w)$ has zero integral with respect 
to $|d w|$ on $\mathbb{T}$. We would like to compute the Fourier series of $w \mapsto D_{f} F(\lambda, 0)(h)(w)$ in terms of the Fourier series of $h$

$$
h(w)=\sum_{n=0}^{\infty} b_{n} \bar{w}^{n}, \quad w \in \mathbb{T} .
$$

Since $h \in X$ the Fourier coefficients $b_{n}$ of $h$ are real. Using the expansion

$$
\log \left(1-\frac{w}{\tau}\right)=-\sum_{n=1}^{\infty} \frac{1}{n} w^{n} \bar{\tau}^{n}
$$

and computing we get

$$
D_{f} F(\lambda, 0)(h)(w)=\sum_{n=1}^{\infty}\left(\lambda-\frac{1}{n}\right) b_{n-1} w^{n}+\sum_{n=1}^{\infty}\left(\lambda-\frac{1}{n}\right) b_{n-1} \bar{w}^{n} .
$$

From the above expression we immediately conclude that the kernel of $D_{f} F(\lambda, 0)$ is non trivial only if $\lambda=1 / m$ for some positive integer $m$. If this is the case, then the kernel is one dimensional and is generated by the function $w \mapsto \bar{w}^{m-1}=1 / w^{m-1}$. It is precisely at this point when we use the fact that the Fourier coefficients of the functions in our space $X$ are real. If the coefficient were complex, we would get a kernel of real dimension 3 . Let us now look at the range of $D_{f} F(\lambda, 0)$ under the assumption that $\lambda=1 / \mathrm{m}$. Clearly

$$
D_{f} F(1 / m, 0)(h)(w)=2 \sum_{n=1}^{\infty}\left(\frac{1}{m}-\frac{1}{n}\right) b_{n-1} \cos (n \theta), \quad w=e^{i \theta} .
$$

Notice that $D_{f} F(1 / m, 0)(h)$ is a function with zero integral and real Fourier coefficients. We have shown in subsection 3.4 that it is in $C^{1+\alpha}(\mathbb{T})$ and thus in the space $Y$ given by (24). The only Fourier frequency missing in the expansion (46) is $m$, so that it looks plausible that a complement of the range of $D_{f} F(1 / m, 0)$ is the one dimensional subspace generated by $\cos (m \theta)$. To prove this we need to show that each $g \in Y$ with an expansion of the form

$$
g(w)=\sum_{n=1, n \neq m}^{\infty} \beta_{n} \bar{w}^{n}+\sum_{n=1, n \neq m}^{\infty} \beta_{n} w^{n} .
$$

with real $\beta_{n}$ is equal to $D_{f} F(1 / m, 0)(h)$ for some $h \in X$. If $h$ is as in (45) with real Fourier coefficients then the equation $D_{f} F(1 / m, 0)(h)=g$ is equivalent to

$$
2\left(\frac{1}{m}-\frac{1}{n}\right) b_{n-1}=\beta_{n}, \quad n=1,2, . .
$$

or, solving for $b_{n}$,

$$
\begin{aligned}
b_{n} & =\frac{m}{2} \frac{n+1}{n+1-m} \beta_{n+1} \\
& =\frac{m}{2} \beta_{n+1}+\frac{m^{2}}{2} \frac{1}{n+1-m} \beta_{n+1} .
\end{aligned}
$$

The solution to $D_{f} F(1 / m, 0)(h)=g$ is

$$
h(w)=\frac{m}{2} w G(w)+\frac{m^{2}}{2} w H(w)
$$

where

$$
G(w)=\sum_{n=1}^{\infty} \beta_{n} \bar{w}^{n}, \quad w \in \mathbb{T}
$$


and

$$
H(w)=\sum_{n=1, n \neq m}^{\infty} \frac{\beta_{n}}{n-m} \bar{w}^{n}, \quad w \in \mathbb{T} .
$$

The function $G$ is in $C^{1+\alpha}(\mathbb{T})$, and then in $X$, because the Cauchy projection

$$
\sum_{n=-\infty}^{\infty} c_{n} w^{n} \mapsto \sum_{n=0}^{\infty} c_{n} w^{n}
$$

preserves the space $C^{1+\alpha}(\mathbb{T})$. This is false for the space $C^{1}(\mathbb{T})$ of continuously differentiable functions on $\mathbb{T}$ (because the Cauchy projection does not preserve $L^{\infty}(\mathbb{T})$ ) and this is why we cannot choose the space $C^{1}(\mathbb{T})$ in the definition of $X$ and $Y$. It still remains to show that $H \in C^{1+\alpha}(\mathbb{T})$, but this is easy. Set

$$
K(w)=\sum_{n=1, n \neq m}^{\infty} \frac{\bar{w}^{n}}{n-m}, \quad w \in \mathbb{T},
$$

so that $K \in L^{2}(\mathbb{T}) \subset L^{1}(\mathbb{T})$ and $H=G * K \in C^{1+\alpha}(\mathbb{T})$.

To apply Crandall-Rabinowitz's Theorem we still have to check the transversality condition, that is, the the second order partial derivative $D_{f \lambda} F(1 / m, 0)$ of $F$ with respect to $f$ and $\lambda$ applied to the function $w \mapsto \bar{w}^{m-1}$ is not in the range of $D_{f} F(1 / m, 0)$. Now $D_{f \lambda} F(1 / m, 0)$ can be identified with a bounded linear mapping from $X$ into $Y$. It is easy to see that

$$
D_{f \lambda} F(1 / m, 0)(h)(w)=2 \operatorname{Re}(h(w) \bar{w}), \quad h \in X .
$$

Hence

$$
D_{f \lambda} F(1 / m, 0)\left(\bar{w}^{m-1}\right)(w)=2 \operatorname{Re}\left(\bar{w}^{m}\right)=2 \cos (m \theta), \quad w=e^{i \theta},
$$

which is not in the range of $D_{f} F(1 / m, 0)$.

Finally one checks easily that $D_{f \lambda} F(\lambda, f)(h)(w)=2 \operatorname{Re}(\Phi(w) \overline{h(w)})$ is a continuous function on $\mathbb{R} \times V$.

3.7. $m$-fold symmetry. We showed in the previous subsections how to apply CrandallRabinowitz's Theorem to the spaces $X$ and $Y$. The conclusion is that, given a positive integer $m$, we have a continuous curve $\left(\lambda_{\xi}, f_{\xi}\right) \in \mathbb{R} \times V$, defined for $\xi$ in some interval of the form $(1 / m-\delta, 1 / m+\delta)$, such that $F\left(\lambda_{\xi}, f_{\xi}\right)=0, \xi \in(1 / m-\delta, 1 / m+\delta)$. Then $\Phi_{\xi}(z)=z+f_{\xi}(z),|z| \geq 1$, is a conformal mapping of $\mathbb{C}_{\infty} \backslash \bar{\triangle}$ into some domain $U_{\xi}$ and $D_{\xi}=\mathbb{C}_{\infty} \backslash \overline{U_{\xi}}$ is a simply connected vortex patch which rotates with angular velocity $\Omega_{\xi}=\left(1-\lambda_{\xi}\right) / 2$. We know that $D_{\xi}$ is a domain with boundary of class $C^{1+\alpha}$, but nothing else can be said about its symmetry properties without further arguments. The $m$-fold symmetry follows by adding a condition to the spaces $X$ and $Y$.

Given $m$, define $X_{m}$ as the subspace of $X$ consisting of those functions $f \in X$ with a Fourier expansion of the type

$$
f(w)=\sum_{n=1}^{\infty} a_{n m-1} \bar{w}^{n m-1}, \quad w \in \mathbb{T} .
$$

If $f$ is in the open unit ball of $X_{m}$ the expansion of the associated conformal mapping $\Phi$ in $\{z:|z| \geq 1\}$ is given by

$$
\Phi(z)=z\left(1+\sum_{n=1}^{\infty} \frac{a_{n m-1}}{z^{n m}}\right) .
$$

This will provide the $m$-fold symmetry of the associated patch, via the relation

$$
\Phi\left(e^{i 2 \pi / m} z\right)=e^{i 2 \pi / m} \Phi(z),|z| \geq 1 .
$$


The space $Y_{m}$ is the subspace of $Y$ consisting of those $g \in Y$ whose Fourier coefficients vanish at frequencies which are not non-zero multiples of $m$. In other words, the Fourier expansion of $g$ is of the type

$$
g(w)=\sum_{n=0}^{\infty} \beta_{n m} 2 \cos (n m \theta), \quad w=e^{i \theta},
$$

with $\beta_{0}=0$ and real $\beta_{n m}$. Notice that, since the generator $w \mapsto \bar{w}^{m-1}$ of the kernel of $D_{f} F(1 / m, 0)$ is in $X_{m}$ for $m \geq 2$, we still have that the dimension of the kernel is 1 . In the same way the codimension of the range of $D_{f} F(1 / m, 0)$ in $Y_{m}$ is 1 .

However to apply Crandall-Rabinowitz's Theorem to $X_{m}$ and $Y_{m}$ one has to check that $F(\lambda, f) \in Y_{m}$ if $f \in X_{m}$. This follows rather easily from work we have already done. One has to observe that the space $A_{m}$ of continuous functions on $\mathbb{T}$ whose Fourier coefficients vanish at frequencies which are not integer multiples of $m$ is an algebra, closed in the space of continuous functions on $\mathbb{T}$. Next we remark that $w \mapsto \Phi^{\prime}(w) \in A_{m}$. We also need the fact that $w \mapsto \frac{w}{\Phi(w)} \in A_{m}$. To show this, set

$$
g(w)=\frac{\Phi(w)}{w}-1, \quad w \in \mathbb{T},
$$

so that $g \in A_{m}$ and $\|g\|_{\infty}<1$. Thus

$$
w \mapsto \frac{w}{\Phi(w)}=\sum_{n=0}^{\infty}(-1)^{n} g^{n}(w) \in A_{m} .
$$

An easy computation gives that $w \mapsto \lambda|\Phi(w)|^{2}$ belongs to $A_{m}$ if $f \in X_{m}$. It remains to show that $w \mapsto S(f)(w)$ is in $A_{m}$. Recall the identities (??) and (??). First, $a_{n} \neq 0$ only for indexes of the form $n=m q-1$ for some positive integer $q$. On the other hand, $A_{n k}$ is the Fourier coefficient corresponding to the frequency $n-k+1$ of the function $\Phi^{\prime}(\tau)(\tau / \Phi(\tau))^{k}$, which is in $A_{m}$. Hence $A_{n k}$ is non-zero only if $n-k+1=m r$ for some integer $r$. Therefore the sum in $k$ is only over indexes which are multiples of $m$. It remains to examine at the Fourier coefficients of $\Phi(w)^{k}$. Now $\Phi(w)=w g(w)$ with $g \in A_{m}$ and so $\Phi(w)^{k}=w^{k} g(w)^{k}$ is also in $A_{m}$ because only indexes $k$ which are multiples of $m$ have to be taken into account.

Therefore we can apply Crandall-Rabinowitz's Theorem to $X_{m}$ and $Y_{m}$ and finally obtain the existence of $m$-fold symmetric V-states for each integer $m \geq 2$.

3.8. Kirchhoff's ellipses. For $m=2$ we obtain the ellipses parametrized by $w \in \mathbb{T} \mapsto$ $w+\xi \bar{w}$. The real number $\xi$ satisfies $-1<\xi<1$ and is a parameter which determines the shape of the ellipse. The ellipse is centered at 0 , has horizontal semi-axis $1+\xi$, and vertical semi-axis $1-\xi$. The function $z \mapsto z+\frac{\xi}{z}$ is the conformal mapping of the exterior on the unit disc onto the exterior of the ellipse. It is instructive to use CrandallRabinowitz's Theorem to prove that these ellipses rotate. We are going to apply the Theorem to the one dimensional spaces $X$, which is generated by $\bar{w}$, and $Y$, generated by $w^{2}+\bar{w}^{2}=2 \cos (2 \theta), w=e^{i \theta}$. Notice that $X$ is the kernel of $D_{f} F(1 / 2,0)$, which then has range $\{0\}$ of codimension 1 in $Y$. Of course we have to check that $F(\lambda, f)$ sends $X$ into $Y$. Take $f(w)=\xi \bar{w}$ with $|\xi|<1$, so that $\Phi(w)=w+\xi \bar{w}$. The term

$$
|\Phi(w)|^{2}=1+\xi^{2}+\xi w^{2}+\xi \bar{w}^{2}
$$

is correct because the constant $1+\xi^{2}$ will disappear when subtracting the mean. We can compute explicitly $S(f)(w)$ using (??) and the remark that the sum in $n$ and $k$ may be 
reversed because the sum in $n$ is finite. We obtain

$$
S(f)(w)=-\sum_{k=1}^{\infty} \frac{\Phi(w)^{k}}{k} \frac{1}{2 \pi i} \int_{|\tau|=1} \overline{\Phi(\tau)} \Phi^{\prime}(\tau) \frac{1}{\Phi(\tau)^{k}} d \tau .
$$

The only term that survives is that corresponding to the index $k=2$ and the result of the integral is $\xi$. Thus

$$
S(f)(w)=-\frac{\xi}{2}(w+\xi \bar{w})^{2}
$$

and

$$
\left.2 \operatorname{Re} S(f)(w)=-\left(\frac{\xi}{2}\left(1+\xi^{2}\right)\left(w^{2}+\bar{w}^{2}\right)\right)+2 \xi^{2}\right) .
$$

Again the constant term will disappear when subtracting the mean and we conclude that $F(\lambda, f) \in Y$.

A final remark is that, strictly speaking, the conclusion of Crandall-Rabinowitz's Theorem is that for some little interval of $\xi$ centered at 0 the associated ellipse rotates. But, of course, that any ellipse satisfies Burbea's equation (13) can be proved directly. It is interesting to notice that, in this example, $\Phi_{\xi}(z)$ is analytic on a neighborhood of $\{z:|z| \geq 1\}$ for each $\xi \in(-1,1)$, and real analytic in $\xi$ for each $z \in \mathbb{T}$. We do not know how general this fact is.

\section{Boundary SMOOTHNESS OF ROTATING VORTEX PATCHES}

In this section we prove our main result, namely that if the bifurcated patch is close enough to the circle where bifurcation takes place, then the boundary of the patch is of class $C^{\infty}$. Before stating the result more formally we remind the reader of the big picture. We called $V$ the set of functions in the unit ball of $C^{1+\alpha}(\mathbb{T})$ with real Fourier coefficients living only at negative frequencies. Each $f \in V$ determines a conformal mapping $\Phi(z)=$ $z+f(z)$ of the complement of the closed unit disc $\bar{\Delta}$ into some domain containing the point at $\infty$. The boundary of the simply connected domain $D=\mathbb{C} \backslash \Phi(\mathbb{C} \backslash \Delta)$ is the Jordan curve $\Phi(\mathbb{T})$ and so, since $\Phi \in C^{1+\alpha}(\mathbb{T})$, the boundary of $D$ is a Jordan curve of class $C^{1+\alpha}$. Burbea's existence Theorem asserts that for each integer $m \geq 2$ there exists a small positive number $a$ and a continuous curve $f(\xi),-a<\xi<a$, taking values in $V$ such that the simply connected domain $D_{\xi}$ associated with $f(\xi)$ is an $m$-fold rotating vortex patch. Since $f(0)=0, D_{0}$ is the open unit disc and one should think that $D_{\xi}$ is a domain close to the disc, for small values of $\xi \in(-a, a)$, in the topology determined by $C^{1+\alpha}(\mathbb{T})$. We claim that if $D_{\xi}$ is close enough to the disc in the topology given by $C^{1}(\mathbb{T})$ then the boundary of $D_{\xi}$ is of class $C^{\infty}$. Later on we will show that if $D_{\xi}$ is close enough to the disc in the topology given by $C^{2}(\mathbb{T})$ then $D_{\xi}$ is also convex.

Theorem 2. For each integer $m \geq 3$, there exists a small positive $\epsilon_{0}=\epsilon_{0}(m)$ such that if $f \in V$ defines an $m$-fold $V$-state $D$ and $\|f\|_{C^{1}(\mathbb{T})}<\epsilon_{0}$, then $D$ has boundary of class $C^{\infty}$.

Outline of the proof. Before plunging into the details we present a sketch of the proof of Theorem 2. Burbea's equation for $V$-states is $F(\lambda, f)(w)=0, w \in \mathbb{T}$, with

$$
F(\lambda, f)(w)=\lambda|w+f(w)|^{2}+2 \operatorname{Re} S(f)(w)-m(\operatorname{Id}+f, \lambda), \quad w \in \mathbb{T},
$$

where $S(f)$ is given by (18) and $m(\operatorname{Id}+f, \lambda)$ by (16). Recall that the reason to subtract $m(\mathrm{Id}+f, \lambda)$ is that the integral of $F(\lambda, f)$ over $\mathbb{T}$ be zero.

In the previous section we have used bifurcation theory to prove that, given an integer $m \geq 2$ and $0<\alpha<1$, there exists a curve of $V$-states passing through $(1 / m, 0)$ and taking 
values in a little neighborhood of $(1 / m, 0)$ in $(0, \infty) \times V$. Since $V$ is contained in $C^{1+\alpha}(\mathbb{T})$ we conclude that the non-trivial $V$-states we have found have boundary of class $C^{1+\alpha}(\mathbb{T})$. The same approach can be adapted with slight modifications to the space $C^{n+\alpha}(\mathbb{T})$, for each positive integer $n$ and each $0<\alpha<1$. This provides curves of solutions with boundaries of class $C^{n+\alpha}$. However the neighborhood of $(1 / m, 0)$ containing the curve of solutions decreases as $n$ increases and so the $C^{\infty}$ regularity of the boundary cannot be reached by using Crandall-Rabinowitz as a black box. Since Crandall-Rabinowitz depends essentially on the implicit function theorem, we thought of resorting to NashMoser implicit function theorem for $C^{\infty}(\mathbb{T})$. Unfortunately we were not able to implement this idea. We realized later that a simpler method works. The idea is to differentiate the equation $F(\lambda, f)(w)=0$ with respect to $w$ and carefully study the resulting equation. We find a surprising smoothing effect for the unit tangent field to the curve $\Phi(\mathbb{T})$ which induces in turn a global smoothing effect for the conformal mapping $\Phi$. To be more precise, we compute $\frac{d F(\lambda, f)}{d w}$, which yields a formula for the quotient

$$
q(w)=\frac{\overline{\Phi^{\prime}(w)}}{\Phi^{\prime}(w)}, \quad w \in \mathbb{T}
$$

of the form

$$
q(w)=w^{2} \frac{(1-\lambda) \overline{\Phi(w)}+I_{1}(w)}{(1-\lambda) \Phi(w)+\overline{I_{1}(w)}}, \quad w \in \mathbb{T} .
$$

where $I_{1}$ is the integral

$$
I_{1}(w)=\frac{1}{2 \pi i} \int_{\mathbb{T}} \overline{\frac{\Phi(\tau)-\Phi(w)}{\Phi(\tau)-\Phi(w)}} \Phi^{\prime}(\tau) d \tau .
$$

As we know, a priori $\Phi \in C^{1+\alpha}(\mathbb{T})$ and thus $q$ is only in $C^{\alpha}(\mathbb{T})$. But (47) suggests that $q$ might be of class $C^{1+\alpha}(\mathbb{T})$ provided $I_{1}(w)$ is. It is not difficult to compute the derivative of $I_{1}$ and check that it is in $C^{\beta}(\mathbb{T}), 0<\beta<\alpha$. Thus we get that $q \in C^{1+\beta}(\mathbb{T}), 0<\beta<\alpha$, if the denominator in (47) does not vanish in $\mathbb{T}$. This is guaranteed by the smallness condition $\|f\|_{C^{1}(\mathbb{T})}<\epsilon_{0}$. Now the smoothness of $q$ is the same as that of the unit tangent vector to the curve $\Phi(\mathbb{T})$ and classical results on the smoothness of conformal mappings yield that $\Phi \in C^{2+\beta}(\mathbb{T}), 0<\beta<\alpha$. One can then iterate the argument and show that under the assumption $\Phi \in C^{2+\beta}(\mathbb{T}), 0<\beta<\alpha$ the function $I_{1}$ can be differentiated twice with respect to $w$ and the second derivative is in $C^{\beta}(\mathbb{T}), 0<\beta<\alpha$. Thus $\Phi \in$ $C^{3+\beta}(\mathbb{T}), 0<\beta<\alpha$. Since the iteration can be performed any number of times we conclude that $\Phi \in C^{\infty}(\mathbb{T})$. We begin now with the details of the proof.

Proof of Theorem Q. To get (47) we differentiate the equation $F(\lambda, f)(w)=0$ with respect to $w$ and use (21). We get

$$
\lambda\left(\Phi^{\prime}(w) \overline{\Phi(w)}-\frac{1}{w^{2}} \overline{\Phi^{\prime}(w)} \Phi(w)\right)+\frac{d S(f)}{d w}(w)-\frac{1}{w^{2}} \frac{\overline{d S(f)}}{d w}(w)=0 .
$$

Recall that the parameter $\lambda$ is taken in the interval ]0,1[. According to (25) and (31),

$$
\begin{aligned}
\frac{d S(f)}{d w}(w) & =-\Phi^{\prime}(w)\left(\overline{\Phi(w)}+\frac{1}{2 \pi i} \int_{\mathbb{T}} \frac{\overline{\Phi(\tau)-\Phi(w)}}{\Phi(\tau)-\Phi(w)} \Phi^{\prime}(\tau) d \tau\right) \\
& :=-\Phi^{\prime}(w)\left(\overline{\Phi(w)}+I_{1}(w)\right), \quad w \in \mathbb{T} .
\end{aligned}
$$

Putting together the two preceding identities and setting $q(w):=\frac{\overline{\Phi^{\prime}(w)}}{\Phi^{\prime}(w)}$ we obtain (47). Let us show that the denominator in (47) does not vanish on $\mathbb{T}$ if $\|f\|_{C^{1}(\mathbb{T})}$ is small enough. 
Since $\Phi(w)=w+f(w), w \in \mathbb{T}$, the denominator in (47) is

$$
D(w)=(1-\lambda)(w+f(w))+\overline{I_{1}(w)}, \quad w \in \mathbb{T} .
$$

Now

$$
\begin{aligned}
I_{1}(w) & =\frac{1}{2 \pi i} \int_{\mathbb{T}} \frac{\overline{\tau-w}}{\Phi(\tau)-\Phi(w)} \Phi^{\prime}(\tau) d \tau+\frac{1}{2 \pi i} \int_{\mathbb{T}} \frac{\overline{f(\tau)-f(w)}}{\Phi(\tau)-\Phi(w)} \Phi^{\prime}(\tau) d \tau \\
& =-\frac{1}{w} \frac{1}{2 \pi i} \int_{\mathbb{T}} \frac{\tau-w}{\Phi(\tau)-\Phi(w)} \Phi^{\prime}(\tau) \frac{d \tau}{\tau}+J_{1}(w) .
\end{aligned}
$$

where $J_{1}$ is a notation for the second term and we have used the identity

$$
\overline{\tau-w}=-\frac{\tau-w}{\tau w}, \quad \tau, w \in \mathbb{T} .
$$

On the other hand, by Lebesgue dominated convergence Theorem we have

$$
\frac{1}{2 \pi i} \int_{\mathbb{T}} \frac{\tau-w}{\Phi(\tau)-\Phi(w)} \Phi^{\prime}(\tau) \frac{d \tau}{\tau}=\lim _{\varepsilon \rightarrow 0} \frac{1}{2 \pi i} \int_{|\tau|=1+\varepsilon} \frac{\tau-w}{\Phi(\tau)-\Phi(w)} \Phi^{\prime}(\tau) \frac{d \tau}{\tau} .
$$

The integral on the circle of radius $1+\varepsilon$ is 1 for each positive $\varepsilon$, because the integrand is an analytic function of $\tau$ in the exterior of the unit disc whose first term in the expansion at $\infty$ is $1 / \tau$. Hence

$$
I_{1}(w)=-\bar{w}+J_{1}(w), \quad w \in \mathbb{T} .
$$

Plugging this identity into (49)

$$
D(w)=-\lambda w+(1-\lambda) f(w)+\overline{J_{1}(w)}, \quad w \in \mathbb{T} .
$$

The estimate of the $L^{\infty}$ norm of $J_{1}$ can be easily performed as follows

$$
\begin{aligned}
\left\|J_{1}\right\|_{L^{\infty}(\mathbb{T})} & \leq \frac{\left\|f^{\prime}\right\|_{L^{\infty}(\mathbb{T})}}{\inf _{\tau \neq w} \frac{|\Phi(\tau)-\Phi(w)|}{|\tau-w|}}\left\|\Phi^{\prime}\right\|_{L^{\infty}(\mathbb{T})} \\
& \leq \frac{\left\|f^{\prime}\right\|_{L^{\infty}(\mathbb{T})}}{1-\left\|f^{\prime}\right\|_{L^{\infty}(\mathbb{T})}}\left(1+\left\|f^{\prime}\right\|_{L^{\infty}}\right) .
\end{aligned}
$$

Therefore

$$
\begin{aligned}
|D(w)| & \geq \lambda-(1-\lambda)\|f\|_{L^{\infty}(\mathbb{T})}-\left\|\tilde{I}_{1}\right\|_{L^{\infty}(\mathbb{T})} \\
& \geq \lambda-\|f\|_{\infty}-\left\|f^{\prime}\right\|_{\infty} \frac{1+\left\|f^{\prime}\right\|_{\infty}}{1-\left\|f^{\prime}\right\|_{\infty}} \\
& \geq \lambda-2 \frac{\|f\|_{C^{1}(\mathbb{T})}}{1-\|f\|_{C^{1}(\mathbb{T})}} \\
& \geq \frac{1}{2} \lambda,
\end{aligned}
$$

where the last inequality holds provided $\|f\|_{C^{1}(\mathbb{T})} \leq \frac{\lambda}{4+\lambda}$.

Let us now prove that the function $w \in \mathbb{T} \mapsto I_{1}(w)$ is more regular than one would expect. Indeed, it belongs to the space $C^{1+\beta}(\mathbb{T})$ for any $\beta$ satisfying $0<\beta<\alpha$. Since the quotient $w \mapsto \frac{\overline{\Phi(\tau)-\Phi(w)}}{\Phi(\tau)-\Phi(w)}$ extends continuously to the diagonal of $\mathbb{T}$, in taking derivatives inside the integral defining $I_{1}(w)$ no "boundary terms" will appear. Then it follows from 
(21) and (31) that

$$
\begin{aligned}
\frac{d I_{1}}{d w}(w) & =\frac{1}{w^{2}} \overline{\Phi^{\prime}(w)} \text { p.v. } \frac{1}{2 \pi i} \int_{\mathbb{T}} \frac{\Phi^{\prime}(\tau)}{\Phi(\tau)-\Phi(w)} d \tau \\
& +\Phi^{\prime}(w) \text { p.v. } \frac{1}{2 \pi i} \int_{\mathbb{T}} \frac{\overline{\Phi(\tau)-\Phi(w)}}{(\Phi(\tau)-\Phi(w))^{2}} \Phi^{\prime}(\tau) d \tau \\
& =\frac{\overline{\Phi^{\prime}(w)}}{2 w^{2}}+\Phi^{\prime}(w) \text { p.v. } \frac{1}{2 \pi i} \int_{\mathbb{T}} \frac{\overline{\Phi(\tau)-\Phi(w)}}{(\Phi(\tau)-\Phi(w))^{2}} \Phi^{\prime}(\tau) d \tau .
\end{aligned}
$$

To obtain the appropriate Hölder estimate we are looking for it is convenient to write the principal value integral above as the sum of two terms by adding and subtracting $\overline{\Phi^{\prime}(w)(\tau-w)}$ in the numerator of the fraction. We get

$$
\begin{aligned}
\text { p.v. } \frac{1}{2 \pi i} \int_{\mathbb{T}} \frac{\overline{\Phi(\tau)-\Phi(w)}}{(\Phi(\tau)-\Phi(w))^{2}} \Phi^{\prime}(\tau) d \tau & =\frac{1}{2 \pi i} \int_{\mathbb{T}} \frac{\overline{\Phi(\tau)-\Phi(w)-\Phi^{\prime}(w)(\tau-w)}}{(\Phi(\tau)-\Phi(w))^{2}} \Phi^{\prime}(\tau) d \tau \\
& +\overline{\Phi^{\prime}(w)} \text { p.v. } \frac{1}{2 \pi i} \int_{\mathbb{T}} \frac{\overline{\tau-w}}{(\Phi(\tau)-\Phi(w))^{2}} \Phi^{\prime}(\tau) d \tau
\end{aligned}
$$

Call $I_{2}(w)$ the first term in the right-hand side above, namely,

$$
I_{2}(w):=\frac{1}{2 \pi i} \int_{\mathbb{T}} \frac{\overline{\Phi(\tau)-\Phi(w)-\Phi^{\prime}(w)(\tau-w)}}{(\Phi(\tau)-\Phi(w))^{2}} \Phi^{\prime}(\tau) d \tau, \quad w \in \mathbb{T} .
$$

Let us compute the principal value integral in the second term of (53). By (50) (54)

$$
\text { p.v. } \frac{1}{2 \pi i} \int_{\mathbb{T}} \frac{\overline{\tau-w}}{(\Phi(\tau)-\Phi(w))^{2}} \Phi^{\prime}(\tau) d \tau=-\frac{1}{w} \text { p.v. } \frac{1}{2 \pi i} \int_{\mathbb{T}}\left(\frac{\tau-w}{\Phi(\tau)-\Phi(w)}\right)^{2} \frac{\Phi^{\prime}(\tau)}{\tau} \frac{d \tau}{\tau-w} .
$$

We compute the principal value integral above by the method used in dealing with (30). Denote by $\gamma_{\epsilon}, \epsilon>0$, the arc which is the intersection of the circle centered at $w$ of radius $\epsilon$ and the complement of the open unit disc, with the counter-clockwise orientation. Let $\mathbb{T}_{\epsilon}$ the closed Jordan curve consisting of the arc $\gamma_{\epsilon}$ followed by the part of the unit circle at distance from $w$ not less than $\epsilon$, traversed counterclockwise. Then the principal value in (154) is

$$
\text { p.v. } \begin{aligned}
\frac{1}{2 \pi i} \int_{\mathbb{T}}\left(\frac{\tau-w}{\Phi(\tau)-\Phi(w)}\right)^{2} \frac{\Phi^{\prime}(\tau)}{\tau} \frac{d \tau}{\tau-w} & =\lim _{\varepsilon \rightarrow 0} \frac{1}{2 \pi i} \int_{\mathbb{T}_{\varepsilon}}\left(\frac{\tau-w}{\Phi(\tau)-\Phi(w)}\right)^{2} \frac{\Phi^{\prime}(\tau)}{\tau} \frac{d \tau}{\tau-w} \\
& -\lim _{\varepsilon \rightarrow 0} \frac{1}{2 \pi i} \int_{\gamma_{\varepsilon}}\left(\frac{\tau-w}{\Phi(\tau)-\Phi(w)}\right)^{2} \frac{\Phi^{\prime}(\tau)}{\tau} \frac{d \tau}{\tau-w} .
\end{aligned}
$$

The integral on $\mathbb{T}_{\epsilon}$ is zero because the integrand is analytic in the exterior of the unit disc and has a double zero at $\infty$. The limit of the integral on $\gamma_{\epsilon}$ is given by

Therefore

$$
\lim _{\varepsilon \rightarrow 0} \frac{1}{2 \pi i} \int_{\gamma_{\varepsilon}}\left(\frac{\tau-w}{\Phi(\tau)-\Phi(w)}\right)^{2} \frac{\Phi^{\prime}(\tau)}{\tau} \frac{d \tau}{\tau-w}=\frac{1}{2 w \Phi^{\prime}(w)} .
$$

$$
\frac{d I_{1}(w)}{d w}=\Phi^{\prime}(w) I_{2}(w)+\frac{\overline{\Phi^{\prime}(w)}}{w^{2}}, \quad w \in \mathbb{T} .
$$

Lemma 3 below applied for $n=2$ yields $I_{2} \in C^{\beta}(\mathbb{T})$, for each $\beta$ satisfying $0<\beta<\alpha$ and hence we conclude that $w \mapsto \frac{d I_{1}}{d w}(w)$ belongs to the space $C^{\beta}(\mathbb{T}), \quad 0<\beta<\alpha$, that is, that $I_{1} \in C^{1+\beta}(\mathbb{T}), 0<\beta<\alpha$. Thus from the expression (47), we find that

$$
q \in C^{1+\beta}(\mathbb{T}), \quad 0<\beta<\alpha .
$$


Before dealing with Lemma 3 we discuss how the smoothness of $q$ translates into the same type of smoothness of $\Phi^{\prime}$. More precisely, we will prove the following: for each positive integer $n$ and $0<\beta<1$,

$$
q \in C^{n+\beta}(\mathbb{T} ; \mathbb{T}) \Longrightarrow \Phi \in C^{n+1+\beta}(\mathbb{T} ; \mathbb{C})
$$

For this purpose, we first relate the regularity of the map $w \mapsto q(w)$ to the smoothness of the Jordan curve $\Phi(\mathbb{T})$ and then we use the Kellogg-Warschawski Theorem [WS] to get a global regularity result for the conformal map $\Phi$.

Using the conformal parametrization $\theta \in \mathbb{R} \mapsto \Phi\left(e^{i \theta}\right)$, we get easily the following formula for the unit tangent vector $\vec{\tau}(\theta)$ to the curve $\Phi(\mathbb{T})$ at the point $\Phi\left(e^{i \theta}\right)$,

$$
\vec{\tau}(\theta)=\frac{\frac{d}{d \theta} \Phi\left(e^{i \theta}\right)}{\left|\frac{d}{d \theta} \Phi\left(e^{i \theta}\right)\right|}=i w \frac{\Phi^{\prime}(w)}{\left|\Phi^{\prime}(w)\right|}, \quad w=e^{i \theta} .
$$

Consequently

$$
[\vec{\tau}(\theta)]^{2}=-w^{2} \overline{q(w)}, \quad w=e^{i \theta} .
$$

Since $\Phi$ belongs to $C^{1+\alpha}$, the map $\theta \mapsto \vec{\tau}(\theta)$ must be in $C^{\alpha}(\mathbb{R} ; \mathbb{T}) \subset C(\mathbb{R} ; \mathbb{T})$ and by the lifting theorem there exists a continuous function $\phi: \mathbb{R} \rightarrow \mathbb{R}$, such that

$$
\vec{\tau}(\theta)=e^{i \phi(\theta)}, \theta \in \mathbb{R} .
$$

Recall that we have established that $q \in C^{1+\beta}(\mathbb{T}), 0<\beta<\alpha$ and so $[\vec{\tau}]^{2}$ remains in the same space. Since the argument function $\phi$ can be recovered by the formula

$$
\phi(\theta)=\phi(0)-\frac{1}{2} i \int_{0}^{\theta} \frac{\sigma^{\prime}(t)}{\sigma(t)} d t, \quad \text { with } \quad \sigma(t):=[\vec{\tau}(t)]^{2},
$$

$\phi$ is in $C^{1+\beta}(\mathbb{R})$ and consequently $\vec{\tau} \in C^{1+\beta}(\mathbb{R})$. More generally, the preceding formula for $\phi$ shows that, for each non-negative integer $n$ and $\beta \in] 0,1[$,

$$
q \in C^{n+\beta}(\mathbb{T} ; \mathbb{T}) \Longrightarrow \vec{\tau} \in C^{n+\beta}(\mathbb{R} ; \mathbb{T}) .
$$

Now we will use the Kellogg-Warschawski theorem [WS], which can be also found in [P, Theorem 3.6]. It asserts that if the boundary $\Phi(\mathbb{T})$ is a Jordan curve of class $C^{n+1+\beta}$, with $n$ a non-negative integer and $0<\beta<1$, then the conformal map $\Phi: \mathbb{C} \backslash \bar{\Delta} \rightarrow \mathbb{C}$ has a continuous extension to $\mathbb{C} \backslash \Delta$ which is of class $C^{n+1+\beta}$. In other words,

$$
\vec{\tau} \in C^{n+\beta}(\mathbb{R} ; \mathbb{T}) \Longrightarrow \Phi \in C^{n+1+\beta}(\mathbb{T} ; \mathbb{C}) .
$$

Combining (56) and (57) we obtain

$$
\Phi \in C^{2+\beta}(\mathbb{T}) .
$$

We are now ready to iterate the preceding argument. Assume that $\Phi \in C^{n-1+\beta}(\mathbb{T})$, $0<\beta<1$, for some $n \geq 3$. We are going to show that,

$$
\Phi \in C^{n+\gamma}(\mathbb{T}), \quad 0<\gamma<\beta .
$$

This will complete the proof that $\Phi \in C^{\infty}(\mathbb{T})$.

We need the following general lemma. For $\Phi \in C^{n}(\mathbb{T})$, let

$$
P_{n}(\Phi)(\tau, w)=\sum_{j=0}^{n} \frac{\Phi^{(j)}(w)}{j !}(\tau-w)^{j}
$$

be the Taylor polynomial of degree $n$ of $\Phi$, around the point $w$, evaluated at the point $\tau$. 
Lemma 3. Assume that $\Phi \in C^{n-1+\alpha}(\mathbb{T})$, for $n \geq 2,0<\alpha<1$. Let $T_{n}$ be the operator

$$
T_{n} g(w)=\frac{1}{2 \pi i} \int_{\mathbb{T}} K_{n}(w, \tau) g(\tau) d \tau, \quad w \in \mathbb{T}, \quad g \in L^{\infty}(\mathbb{T})
$$

with kernel

Then, for any $\beta$ satisfying $0<\beta<\alpha$,

$$
K_{n}(w, \tau)=\frac{\overline{\Phi(\tau)-P_{n-1}(\Phi)(\tau, w)}}{(\Phi(\tau)-\Phi(w))^{n}} .
$$

$$
\left\|T_{n} g\right\|_{C^{\beta}(\mathbb{T})} \leq C\|g\|_{L^{\infty}(\mathbb{T})}
$$

Proof. The lemma is easily proven by standard methods, as in [MOV, p.419], once one knows that $K_{n}$ satisfies

$$
\left|K_{n}(w, \tau) \leq C\right| \tau-\left.w\right|^{\alpha-1}, \quad \tau, w \in \mathbb{T}, \tau \neq w
$$

and

$$
\left|K_{n}\left(w_{1}, \tau\right)-K_{n}\left(w_{2}, \tau\right)\right| \leq C \frac{\left|w_{1}-w_{2}\right|^{\alpha}}{\left|\tau-w_{1}\right|}, \quad w_{1}, w_{2} \in \mathbb{T},\left|\tau-w_{1}\right| \geq 2\left|w_{1}-w_{2}\right| .
$$

It is obvious that (61) holds by Taylor's formula. For (62) we write

$$
\begin{aligned}
\left|K_{n}\left(w_{1}, \tau\right)-K_{n}\left(w_{2}, \tau\right)\right| & \leq\left|\frac{\overline{P_{n-1}(\Phi)\left(\tau, w_{1}\right)}-\overline{P_{n-1}(\Phi)\left(\tau, w_{2}\right)}}{\left(\Phi(\tau)-\Phi\left(w_{1}\right)\right)^{n}}\right| \\
& +\left|\Phi(\tau)-P_{n-1}(\Phi)\left(\tau, w_{2}\right)\right|\left|\frac{1}{\left(\Phi(\tau)-\Phi\left(w_{1}\right)\right)^{n}}-\frac{1}{\left(\Phi(\tau)-\Phi\left(w_{2}\right)\right)^{n}}\right| \\
& :=\mathrm{I}+\mathrm{II} .
\end{aligned}
$$

The term II can easily be controlled via a gradient estimate by

$$
\begin{aligned}
|\mathrm{II}| & \leq C\left|\tau-w_{2}\right|^{n-1+\alpha} \frac{\left|w_{1}-w_{2}\right|}{\left|\tau-w_{1}\right|^{n+1}} \\
& \leq C \frac{\left|w_{1}-w_{2}\right|^{\alpha}}{\left|\tau-w_{1}\right|} .
\end{aligned}
$$

We have used in the last inequality the equivalence $\frac{1}{2}\left|\tau-w_{1}\right| \leq\left|\tau-w_{2}\right| \leq \frac{3}{2}\left|\tau-w_{1}\right|$. The term $I$ is estimated by observing that there is an elementary formula for the difference of two Taylor's polynomials around different points $w_{1}$ and $w_{2}$, namely,

(63) $P_{n-1}(\Phi)\left(\tau, w_{2}\right)-P_{n-1}(\Phi)\left(\tau, w_{1}\right)=\sum_{j=0}^{n-1}\left(\Phi^{(j)}\left(w_{2}\right)-P_{n-1-j}\left(\Phi^{(j)}\right)\left(w_{2}, w_{1}\right)\right) \frac{\left(\tau-w_{2}\right)^{j}}{j !}$.

This follows easily from the identity,

$$
\begin{aligned}
P_{n-1}(\Phi)\left(\tau, w_{1}\right) & =\sum_{j=0}^{n-1} \frac{\left(\tau-w_{2}\right)^{j}}{j !}\left\{\partial_{\tau}^{(j)}\left[P_{n-1}(\Phi)\right]\right\}\left(w_{2}, w_{1}\right) \\
& =\sum_{j=0}^{n-1} \frac{\left(\tau-w_{2}\right)^{j}}{j !} \sum_{k=j}^{n-1} \frac{\left(w_{2}-w_{1}\right)^{k-j}}{(k-j) !} \Phi^{(k)}\left(w_{1}\right) \\
& =\sum_{j=0}^{n-1} \frac{\left(\tau-w_{2}\right)^{j}}{j !} P_{n-1-j}\left(\Phi^{(j)}\right)\left(w_{2}, w_{1}\right) .
\end{aligned}
$$

Since $\Phi^{(j)}$ belongs to $C^{n-1-j+\alpha}(\mathbb{T})$ then

$$
\left|\Phi^{(j)}\left(w_{2}\right)-P_{n-1-j}\left(\Phi^{(j)}\right)\left(w_{2}, w_{1}\right)\right| \leq C\left|w_{2}-w_{1}\right|^{n-1-j+\alpha} .
$$


Combining this estimate with formula (63) yields

$$
\begin{aligned}
|\mathrm{I}| & \leq C \sum_{j=0}^{n-1}\left|w_{2}-w_{1}\right|^{n-1-j+\alpha} \frac{\left|\tau-w_{2}\right|^{j}}{j !} \frac{1}{\left|\tau-w_{1}\right|^{n}} \\
& \leq C \frac{\left|w_{1}-w_{2}\right|^{\alpha}}{\left|\tau-w_{1}\right|} .
\end{aligned}
$$

In view of (47) the only task left is to show that $I_{1} \in C^{n-1+\gamma}(\mathbb{T}), 0<\gamma<\beta$, provided $\Phi \in C^{n-1+\beta}(\mathbb{T})$. Indeed, this will lead to $q \in C^{n-1+\gamma}(\mathbb{T})$ and, according to the discussion above on the Kellog-Warschawski Theorem, we conclude that $\Phi \in C^{n+\gamma}(\mathbb{T})$. Now, in order to prove that $I_{1} \in C^{n-1+\gamma}(\mathbb{T})$ we need to establish a recursive formula for the higher order derivatives of $I_{1}$, which is the goal of the next lemma.

Lemma 4. Let $\Phi \in C^{n+\beta}(\mathbb{T}), 0<\beta<1, n \geq 2$, and set

$$
I_{n}(w):=\frac{1}{2 \pi i} \int_{\mathbb{T}} \frac{\overline{\Phi(\tau)-P_{n-1}(\Phi)(\tau, w)}}{(\Phi(\tau)-\Phi(w))^{n}} \Phi^{\prime}(\tau) d \tau, \quad w \in \mathbb{T} .
$$

Then

$$
\frac{d I_{n}(w)}{d w}=n \Phi^{\prime}(w) I_{n+1}(w), w \in \mathbb{T} .
$$

Remark. Notice that formula (51) for the derivative of $I_{1}$ falls out of the scope of (64). Indeed, it is a fortunate fact that a compact formula as (64) can be found.

The proof of the preceding lemma depends on the following calculation.

Sublemma. Let $\Phi \in C^{1}(\mathbb{T})$ and $n \geq 1$. Then

$$
\text { p.v. } \frac{1}{2 \pi i} \int_{\mathbb{T}} \frac{(\tau-w)^{n}}{(\Phi(\tau)-\Phi(w))^{n+1}} \Phi^{\prime}(\tau) \frac{d \tau}{\tau^{n}}=-\frac{1}{2 \Phi^{\prime}(w)^{n} w^{n}}, \quad w \in \mathbb{T} .
$$

Proof. Consider again the closed Jordan curve $\mathbb{T}_{\epsilon}$ and the arc $\gamma_{\epsilon}$ used to deal with (30) and (54). The principal value integral in the statement of the sublemma is the limit, as $\epsilon$ tends to 0 , of the sum of two terms. The first is the integral over $\mathbb{T}_{\epsilon}$ of the integrand in (65), which is zero because the integrand is an analytic function of $\tau$ in the exterior of the unit disc with a zero at $\infty$ of order at least 2 . The second term is minus the limit as $\epsilon$ tends to 0 of the integral of the same expression over the arc $\gamma_{\epsilon}$. Since

$$
\frac{1}{2 \pi i} \lim _{\epsilon \rightarrow 0} \int_{\gamma_{\epsilon}} \frac{\Phi^{\prime}(\tau)}{\Phi(\tau)-\Phi(w)} d \tau=\frac{1}{2 \pi i} \lim _{\epsilon \rightarrow 0} \Delta_{\gamma_{\epsilon}} \log \Phi=\frac{1}{2}
$$

it is clear that the limit of the second term is $-1 /\left(2 \Phi^{\prime}(w)^{n} w^{n}\right)$. Here $\Delta_{\gamma_{\epsilon}} \log \Phi$ stands for the variation of $\log \Phi$ on the $\operatorname{arc} \gamma_{\epsilon}$.

Proof of Lemma 4. Since $\Phi \in C^{n}(\mathbb{T})$, the fraction in the integrand in $I_{n}$ extends continuously to the diagonal of $\mathbb{T}$ taking the value $(-1)^{n} \overline{\Phi^{(n)}(w)} \bar{w}^{2 n} /\left(n ! \Phi^{\prime}(w)^{n}\right)$. We can then take derivatives inside the integral and the boundary terms arising in the integration by 
parts are zero. Thus

$$
\begin{aligned}
\frac{d I_{n}}{d w}(w) & =n \Phi^{\prime}(w) \text { p.v. } \frac{1}{2 \pi i} \int_{\mathbb{T}} \frac{\overline{\Phi(\tau)-P_{n-1}(\Phi)(\tau, w)}}{(\Phi(\tau)-\Phi(w))^{n+1}} \Phi^{\prime}(\tau) d \tau \\
& +\frac{1}{n-1 !} \frac{\overline{\Phi^{(n)}(w)}}{w^{2}} \text { p.v. } \frac{1}{2 \pi i} \int_{\mathbb{T}} \frac{(\overline{\tau-w})^{n-1}}{(\Phi(\tau)-\Phi(w))^{n}} \Phi^{\prime}(\tau) d \tau \\
& :=T_{1}(w)+T_{2}(w) .
\end{aligned}
$$

The next move consists in adding and subtracting $\overline{\Phi^{(n)}(w)(\tau-w)^{n}} / n$ ! to the numerator of the fraction in the integrand of $T_{1}(w)$. The result is

$$
\begin{aligned}
T_{1}(w) & =n \Phi^{\prime}(w) \mathrm{p} . \mathrm{v} \cdot \frac{1}{2 \pi i} \int_{\mathbb{T}} \frac{\overline{\Phi(\tau)-P_{n}(\Phi)(\tau, w)}}{(\Phi(\tau)-\Phi(w))^{n+1}} \Phi^{\prime}(\tau) d \tau \\
& +\Phi^{\prime}(w) \frac{\overline{\Phi^{(n)}(w)}}{n-1 !} \mathrm{p} \cdot \mathrm{v} \cdot \frac{1}{2 \pi i} \int_{\mathbb{T}} \frac{(\overline{\tau-w})^{n}}{(\Phi(\tau)-\Phi(w))^{n+1}} \Phi^{\prime}(\tau) d \tau \\
& =n \Phi^{\prime}(w) I_{n+1}(w)+\Phi^{\prime}(w) \frac{\overline{\Phi^{(n)}(w)}}{n-1 !} \mathrm{p} \cdot \mathrm{v} \cdot \frac{1}{2 \pi i} \int_{\mathbb{T}} \frac{(\overline{\tau-w})^{n}}{(\Phi(\tau)-\Phi(w))^{n+1}} \Phi^{\prime}(\tau) d \tau \\
& :=n \Phi^{\prime}(w) I_{n+1}(w)+T_{3}(w)
\end{aligned}
$$

We claim that $T_{2}(w)+T_{3}(w)=0$, which ends the proof of the lemma. The only difficulty is to compute the principal value integrals, which are the same except for a shift in the exponents of the integrand. For instance, by (50) and the sublemma we see that the principal value integral in the term $T_{3}(w)$ is

$$
\begin{aligned}
\text { p.v. } \frac{1}{2 \pi i} \int_{\mathbb{T}} \frac{(\overline{\tau-w})^{n}}{(\Phi(\tau)-\Phi(w))^{n+1}} \Phi^{\prime}(\tau) d \tau & =\frac{(-1)^{n}}{w^{n}} \mathrm{p} \cdot \mathrm{v} \cdot \frac{1}{2 \pi i} \int_{\mathbb{T}} \frac{(\tau-w)^{n}}{(\Phi(\tau)-\Phi(w))^{n+1}} \Phi^{\prime}(\tau) \frac{d \tau}{\tau^{n}} \\
& =\frac{(-1)^{n+1}}{2\left[\Phi^{\prime}(w)\right]^{n} w^{2 n}} .
\end{aligned}
$$

This completes the proof of the Lemma 4

We can now finish the proof of Theorem 2. Recall that our assumption is $\Phi \in$ $C^{n-1+\beta}(\mathbb{T})$, where $0<\beta<1$ and $n \geq 3$, and we want to conclude that $I_{1} \in C^{n-1+\gamma}(\mathbb{T})$, $0<\gamma<\beta$. We have already seen that this will give $\Phi \in C^{n+\gamma}(\mathbb{T}), 0<\gamma<\beta$. We apply Lemma 3 and we get that $I_{n}=T_{n}\left(\Phi^{\prime}\right)$ belongs to $C^{\gamma}(\mathbb{T}), 0<\gamma<\beta$. Formula (64) in Lemma 4 readily yields $I_{n-1} \in C^{1+\gamma}(\mathbb{T})$. Iterating the use of (64) we obtain $I_{2} \in C^{n-2+\gamma}(\mathbb{T})$, and so, finally, by (151),$I_{1} \in C^{n-1+\gamma}(\mathbb{T})$.

We end the paper with the following remark.

Corollary. For each integer $m \geq 3$, there exists a small positive $\epsilon_{0}=\epsilon_{0}(m)$ such that if $f \in V$ defines an $m$-fold $V$-state $D,\|f\|_{C^{1}(\mathbb{T})}<\epsilon_{0}$ and $\|f\|_{C^{2}(\mathbb{T})}<1 / 2$, then $D$ is convex.

Proof. As it is well-known, if $D$ is a Jordan domain bounded by a smooth Jordan curve of class $C^{2}$, then $D$ is convex if and only if the curvature of the boundary curve does not change sign on the curve. By Theorem 2 we know that the boundary of our $V$-state $D$ is of class $C^{\infty}$. To compute the curvature at boundary points we resort to the conformal parametrization $\Phi\left(e^{i \theta}\right)$. The velocity vector (or tangent vector) and the principal normal 
to the curve at the point $\Phi(w), w=e^{i \theta}$, are given by

$$
\vec{v}(\theta)=i w \Phi^{\prime}(w) \quad \text { and } \quad \vec{n}(\theta)=-\frac{w \Phi^{\prime}(w)}{\left|\Phi^{\prime}(w)\right|}
$$

respectively. On the other hand, according to a well-known classical formula for the curvature $\kappa(\theta)$ at the point $\Phi\left(e^{i \theta}\right)$, we have

$$
\kappa(\theta)=\frac{\operatorname{Re}\left(d_{\theta}^{2}\left\{\Phi\left(e^{i \theta}\right)\right\} \overline{\vec{n}(\theta)}\right)}{|\vec{v}(\theta)|^{2}} .
$$

A straightforward computation yields

$$
d_{\theta}^{2}\left\{\Phi\left(e^{i \theta}\right)\right\}=-w\left(\Phi^{\prime}(w)+w \Phi^{\prime \prime}(w)\right) .
$$

Thus the curvature is

Since $\Phi(w)=w+f(w)$

$$
\kappa(\theta)=\frac{1}{\left|\Phi^{\prime}(w)\right|} \operatorname{Re}\left(1+w \frac{\Phi^{\prime \prime}(w)}{\Phi^{\prime}(w)}\right) .
$$

and so

$$
1+w \frac{\Phi^{\prime \prime}(w)}{\Phi^{\prime}(w)}=1+w \frac{f^{\prime \prime}(w)}{1+f^{\prime}(w)}
$$

$$
\operatorname{Re}\left(1+w \frac{\Phi^{\prime \prime}(w)}{\Phi^{\prime}(w)}\right) \geq 1-\frac{\left|f^{\prime \prime}(w)\right|}{1-\left|f^{\prime}(w)\right|} \geq 1-\frac{\|f\|_{C^{2}(\mathbb{T})}}{1-\|f\|_{C^{2}(\mathbb{T})}}
$$

which is non-negative if $\|f\|_{C^{2}(\mathbb{T})}<1 / 2$.

The reader will find interesting information on conformal mappings and convexity in Duren's book [Du].

Acknowledgements. J. Mateu and J. Verdera are grateful to J.A. Carrillo for many enlightening conversations on fluid mechanics and to L. Vega for suggesting that one should look at the special nature of the equation to obtain our regularity result. They also acknowledge generous support from the grants 2009SGR420 (Generalitat de Catalunya) and MTM2010-15657 (Ministerio de Ciencia e Innovación).

\section{REFERENCES}

[A] H. Aref, Integrable, chaotic, and turbulent vortex motion in two-dimensional flows, Ann. Rev. Fluid Mech., 15 (1983), 345-389.

[BM] A.L. Bertozzi and A.J. Majda, Vorticity and Incompressible Flow, Cambridge texts in applied Mathematics, Cambridge University Press, Cambridge, (2002).

[B] J. Burbea, Motions of vortex patches, Lett. Math. Phys. 6 (1982), 1-16.

[CR] M. G. Crandall and P.H. Rabinowitz, Bifurcation from simple eigenvalues, J. of Func. Analysis 8 (1971), 321-340.

[DZ] G.S. Deem and N. J. Zabusky, Vortex waves : Stationary "V-states", Interactions, Recurrence, and Breaking, Phys. Rev. Lett. 4013 (1978), 859-862.

[Ch] J.Y. Chemin, Fluides parfaits incompressibles, Astérisque 230, Société Mathématique de France (1995).

[Du] P. L. Duren, Univalent functions, Grundlehren der mathematischen Wissenschaften 259. Springer-Verlag, New York, 1983.

[Ga] A. E. Gatto, On the boundedness on inhomogeneous Lipschitz spaces of fractional integrals, singular integrals and hypersingular integrals associated to non-doubling measures on metric spaces, Collect. Math. 60 (2009), 101-114.

[HHMV] T.Hmidi, F. de la Hoz, J.Mateu and J.Verdera, Doubly connected V-states for the planar Euler equations, arXiv:1409.7096.

[MOV] J. Mateu, J. Orobitg and J. Verdera, Extra cancellation of even Calderón-Zygmund operators and quasiconformal mappings, J. Math. Pures Appl. 91 (4)(2009), 402-431. 
[L] H. Lamb, Hydrodynamics, Dover Publications, New York, (1945).

[P] Ch. Pommerenke, Boundary behaviour of conformal maps, Springer-Verlag, Berlin, 1992.

[V] J. Verdera, $L^{2}$ boundedness of the Cauchy Integral and Menger curvature, Contemporary Mathematics 277 (2001), 139-158.

[WS] S. E. Warschawski, On the higher derivatives at the boundary in conformal mapping, Trans. Amer. Math. Soc. 38 (1935), no. 2, 310-340.

[W] R. Wittmann, Application of a Theorem of M.G. Krein to singular integrals, Trans. Amer. Math. Soc. 299(2) (1987), 581-599.

[WOZ] H.M. Wu, E.A. Overman II and N.J. Zabusky Steady-state solutions of the Euler equations in two dimensions : rotating and translating $V$-states with limiting cases I. Algorithms ans results, J. Comput. Phys. 53 (1984), 42-71.

IRMaR, Université de Rennes 1, Campus de Beaulieu, 35042 Rennes Cedex, France

E-mail address: thmidi@univ-rennes1.fr

Departament de Matemàtiques, Universitat Autònoma de Barcelona, 08193 Bellaterra, Barcelona, Catalonia

E-mail address: mateu@mat.uab.cat

Departament de Matemàtiques, Universitat Autònoma de Barcelona, 08193 Bellaterra, Barcelona, Catalonia

E-mail address: jvm@mat.uab.cat 